\title{
Event-Triggered Compound Learning Tracking Control of Nonstrict-Feedback Nonlinear Systems in Sensor-to-Controller Channel
}

\section{Yingjie Deng ( $\nabla$ dyj_dl@126.com )}

Yanshan University, School of Mechanical Engineering https://orcid.org/0000-0003-0819-9619

Tao Ni

Yanshan University, School of Vehicle and Energy

Jiantao Wang

Yanshan University, School of Vehicle and Energy

\section{Research Article}

Keywords: Event-triggered control, Fuzzy logic system (FLS), Compound learning, Jumps of virtual control laws, Command filter backstepping

Posted Date: April 6th, 2021

DOl: https://doi.org/10.21203/rs.3.rs-372665/v1

License: (c) (i) This work is licensed under a Creative Commons Attribution 4.0 International License.

Read Full License

Version of Record: A version of this preprint was published at Nonlinear Dynamics on October 4th, 2021. See the published version at https://doi.org/10.1007/s11071-021-06870-w. 


\title{
Event-Triggered Compound Learning Tracking Control of Nonstrict-Feedback Nonlinear Systems in Sensor-to-Controller Channel
}

\author{
Yingjie Deng · Tao Ni · Jiantao Wang
}

Received: xx March 2021 / Accepted: xx xx 2021

\begin{abstract}
This paper investigates the event-triggered tracking control of the nonstrict-feedback nonlinear system with the time-varying disturbances. While the fuzzy logic systems (FLSs) serve as the approximators to the unknown dynamics, the compound disturbance is comprised of the time-varying disturbance and the approximation error of the FLS. An event-triggered compound learning algorithm is originally developed to accurately estimate the total uncertainties. By referring to an event-triggered adaptive model, the control laws are derived without provoking the problem of "algebraic loop", seeing Remark 3. The command filters are employed to generate the continuous substitutes for both the virtual control laws and their derivatives, so as to solve the recently proposed problem of "jumps of virtual control laws" arising in the backstepping-based eventtriggered control (ETC) that functions in the channel of sensor to controller. The triggering condition is constructed to guarantee the similarity between the adaptive model and the original system. While the satisfactory learning performance of the FLSs and the compound disturbances estimation are maintained, the proposed control scheme can guarantee the semi-globally uniformly ultimate boundedness (SGUUB) of all the tracking errors. Finally, a numerical experiment is carried out to exemplify the effectiveness of the proposed control scheme.
\end{abstract}

Yingjie Deng

School of Mechanical Engineering, Yanshan University,

Qinhuangdao 066044, China

E-mail: dyj@ysu.edu.cn

Tao Ni (西) · Jiantao Wang

School of Vehicle and Energy, Yanshan University,

Qinhuangdao 066044, China

E-mail: nitao@jlu.edu.cn,wjt@ysu.edu.cn
Keywords Event-triggered control · Fuzzy logic system (FLS) · Compound learning · Jumps of virtual control laws · Command filter backstepping

\section{Introduction}

Plenty of industrial plants can be described by nonstrictfeedback nonlinear systems, such as the ball and beam system, the spring damper system, the remote manipulator, and the stirred tank reactor [1]. As the strictfeedback system is attributed to the special case of the nonstrict-feedback system, the control strategies for the nonstrict-feedback system can easily extend to the strict-feedback one, but conversely, the conclusion is usually not true. It is because in the backstepping framework to control the strict-feedback system, only the states before the current step are required to construct the virtual control laws, whereas all the states are required for this purpose to control the nonstrict-feedback system such that the "algebraic loop" problem emerges [2]. Based on the monotonously increasing property of the bounding function of unknown dynamics, [3,4] developed a so-called variable separation method, in which the unknown dynamics can be bounded by the sum of tracking errors and the backstepping design was available. This method was further combined with the observer design in [5], the small-gain theorem to deal with the unmodeled dynamics in [6], and extended to the stochastic nonstrict-feedback system in [7-9]. To exempt the restrictive assumptions of the unknown dynamics in these literature, [2] fabricated the novel backsteppingbased control laws and parameter adaptation laws, in which the bounded property of fuzzy basis functions was utilized. This method was applied to the switched nonstrict-feedback systems in $[1,10]$, and combined with 
the finite-time control in [11]. Morever, the problem of "complexity explosion" to differentiate the virtual control laws was avoided by introducing the filters in $[10,11]$. By using the semirecurrent neural network$\mathrm{s}$ (NNs) as the one-step predictors, [12] designed the backstepping-based control laws for the discrete nonstrictfeedback system, which solved both the "algebraic loop" problem and the "noncausality" problem [13]. Also based on the property of bounded neural basis functions, [14] integrated the reinforcement learning to the backstepping approach of the discrete nonstrict-feedback system, such that the suboptimal control performance can be achieved, and yet the "noncausality" problem remained to be unsolved.

Two drawbacks can be found in the above attempt$\mathrm{s}$ to control the nonstrict-feedback systems. First, although the FLSs and the NNs were employed as approximators, the update laws of their weights were fabricated by following the principle of " direct adaptive control" [15], such that the closed-loop stability was ensured but the interpretability for approximation was missed. Second, the time-varying disturbances were seldom addressed in these literature. Although [1] considered the disturbances in the system, they were not handled in the control design and the stability analysis utilized the bounded property of them. To overcome the first drawback, we can get enlightened from the "composite learning" technique. To our comprehension, the composite learning adopts the thought to combine "direct adaptive control" with "indirect adaptive control". By referring to a serial-parallel model [16-23] or a predictor $[24,25]$, the identification components (namely the prediction errors) are added to the update laws following "direct adaptive control", such that both the tracking performance and the approximation performance are ensured through the entire control frame. It should be noted that the update laws in [16-25] only utilized the current information of the states, such that the parameters were not convergent if the persistent excitation (PE) condition was not satisfied. To release this constraint, [26-30] utilized the online-recorded data to construct the prediction errors, which leaded to a more relaxed condition of the interval excitation (IE) and the faster parameter convergence. For the second drawback, [20] found that the existence of disturbances in the nonlinear system will degrade the learning performance of the NNs or the FLSs, and neither the unknown dynamics nor the disturbances can be accurately approximated separately, whereas the sum of them can be estimated with a satisfactory precision by combining the composite learning with the disturbance observers. This method was applied in the canonical nonlinear system in [20,21], and the strict-feedback systems in [30].
To be distinguished from the composite learning, the thought to identify both the unknown dynamics and the time-varying disturbances was named as "compound learning" in [30]. While reviewing these literature, we found that the compound learning was never applied to the nonstrict-feedback system. Although [22] applied the composite learning to the nonstrict-feedback system without the disturbances, it followed the primitive predictor-based composite learning. Moreover, it was observed from the above literature that both the compound learning and the composite learning were never achieved in the event-triggered manner.

Another concern of this paper rests with the ETC. As one of the hotspots in the control field, ETC has attracted compelling interests in recent years. Due to its convincible merit to save the communication load, ETC has been widely employed in the strict-feedback systems [31-39], the nonstrict-feedback systems [40-45], and the other industrial plants $[46-53]$. It can be found that $[31-35,40-44,46,49-51]$ only addressed the ETC in the channel of controller to actuator, namely the real control inputs kept unchanged by the zero order holder $(\mathrm{ZOH})$ during the inter-event time and replaced by the command control signals at the triggering instants. As the communication congestion is more apt to occur in the channel of sensor to controller, the ETC in the channel of sensor to controller is indeed more preferred in control practice. For this goal, we notice that the problem of "jumps of virtual control laws" arises while the backstepping design is employed. A brief description of this problem is: while the states are involved in the virtual control law, the renewal of states at the triggering instants will precipitate the virtual control law into a sudden jump. This problem was first put forward in [52], and the formulaic definition will be presented in Section II of this paper. Although [46-48] addressed the backstepping-based ETC in the channel of sensor to controller, this problem did not emerge as the backstepping therein was only used in one step. Thus, it is more meaningful to discuss this problem in the strictfeedback and the nonstrict-feedback systems, in which the backstepping is iteratively used. In [37-39, 45], this problem was neglected and the virtual control laws were deemed to be continuous at the triggering instants. Although the backstepping-based ETC in the channel of sensor to controller was derived therein, it cannot procure a strict analysis on the closed-loop stability. To our knowledge, the problem of "jumps of virtual control laws" was only solved in $[36,52,53]$. In [36], the final control input was constructed as the composition of each virtual control laws without the involvement of event-triggered samples, and approximated by a NN. Although this paper meritoriously solve the problem of 
"jumps of virtual control laws" and circumvented the recursive appearance of virtual control laws in the triggering condtion, the control performance therein totally relied on the approximation of the $\mathrm{NN}$ in the final control input. Because the direct adaptive control was adopted, the learning performance of the NN was not well. $[52,53]$ substituted the virtual control laws with the continuous variables generated by the first-order filters. This setup can solve both the problems of "jumps of virtual control laws" and "complexity explosion". Nevertheless, the continuous variables were not smooth, and a complicated analysis on the parameter selection is required if these schemes are applied in the high-order nonlinear systems.

Motivated by the above challenge, this paper develops an ETC compound learning scheme of the nonstrictfeedback nonlinear system. Based on an event-triggered adaptive fuzzy model, the backstepping-based control laws are derived. The prediction errors are constructed according to the adaptive model and the original system, which are involved in the update laws of the estimates of the weights of FLSs and the compound disturbances. By using the online-recorded data collected during the inter-event time, the update laws are renewed at the triggering instants and kept by the $\mathrm{ZOH}$ during the inter-event time. By referring to the secondorder command filter, the smooth substitute for the virtual control law and the continuous substitute for its derivative are involved in the backstepping design. The adaptive triggering condition is fabricated to ensure the similarity between the adaptive model and the original system. Compared with the precedents, the contributions of this paper are mainly threefold.

1. By referring to the event-triggered adaptive model, the "algebraic loop" problem is avoided in the proposed scheme. Both the control design and the analysis are less complicated than the existing techniques.

2. By using the online-recorded-data-based prediction errors, the event-triggered compound learning technique is originally developed in this paper. Compared with the continuous composite learning schemes, it ensures the better understanding of total uncertainties and less communication resources.

3. By referring to the second-order command filters, the problem of "jumps of virtual control laws" is solved. Moreover, the problem of "complexity explosion" is avoided. Compared with the $[52,53]$ using the first-order filters, the proposed scheme can address the high-order nonlinear system with less complicated analysis.

The remainder of this paper are organized as follows. Section II provides the preliminaries. Section III presents the control design. Section IV designs the triggering condition and analyzes the closed-loop stability of the system. A practical example is provided to exemplify the effectiveness of the proposed scheme in Section V. Section VI concludes the entire work. Appendices proves the bounded estimation errors and Lemma 2.

Uniformly in this paper, $\|\cdot\|$ denotes the Euclidean norm of the vector, $\lambda_{\max }(\cdot)$ denotes the largest eigenvalue of the matrix, $\hat{s}$ denotes the estimate of $s$ and $\tilde{s}=$ $s-\hat{s}$ denotes the estimation error. $t_{j}$ implies the triggering instant with $j=0,1, \cdots,+\infty$, in which $t_{0}$ is also deemed as the initial time. $V^{+}\left(t_{j}\right)$ denotes $\lim _{t \rightarrow t_{j}} V(t)$ from the right side, $\Delta V=V^{+}\left(t_{j}\right)-V\left(t_{j}\right)$ and $\Theta V=$ $V\left(t_{j+1}\right)-V\left(t_{j}\right)$.

\section{Preliminaries}

\subsection{Problem Formulation}

Consider the single-input-single-output (SISO) nonstrictfeedback nonlinear system as

$\left\{\begin{array}{l}\dot{x}_{i}=f_{i}(x)+x_{i+1}+d_{i}, i=1, \cdots, n-1 \\ \dot{x}_{n}=f_{n}(x)+u+d_{n} \\ y=x_{1}\end{array}\right.$

where $x=\left[x_{1}, \cdots, x_{n}\right]^{\mathrm{T}}$ denotes the $\mathrm{n}$-dimensional vector of states, $y$ is the single output, $u$ denotes the single control input, $f_{i}(x)$ denotes the smooth unknown dynamics in each hierarchy and $d_{i}$ denotes the unknown time-varying disturbance. To facilitate the following design and analysis, an assumption is preset as

Assumption $1 \quad[2,20,30,36]$ The time-varying disturbance $d_{i}$ and its first-order derivative $\dot{d}_{i}$ are deemed to be bounded by $\left|d_{i}\right| \leq b_{d_{i}}$ and $\left|\dot{d}_{i}\right| \leq b_{\dot{d}_{i}}$ respectively, where $b_{d_{i}}$ and $b_{\dot{d}_{i}}$ are two unknown positive constants.

Remark 1 The nonstrict-feedback nonlinear system of (1) has the same structure with that in [1]. Without the disturbances, it has the same structure with those in $[5,11,45]$. By setting the gain function as $g_{n}(\cdot)=1$ and reexpressing $f_{i}(\cdot)+g_{i}(\cdot)-x_{i}$ as $f_{i}(x)$, the nonstrictfeedback nonlinear sytems in $[2-4,10,22,42-44]$ and all the referenced strict-feedback systems in this paper are equivalent to (1).

Then, the control objective of this paper is conclude as: design the control law of $u$ such that the output $y$ can track its smooth reference of $y_{d}$, which has the bounded first-order derivative.

Next, we will discuss the problem of "jumps of virtual control laws". Following the backstepping design, the virtual control laws for (1) at the $i$ th step usually 
has the form of $\alpha_{i}=h_{i}\left(\bar{x}_{i}, t\right)$, where $\bar{x}_{i}=\left[x_{1}, \cdots, x_{i}\right]^{\mathrm{T}}$ and $h_{i}\left(\bar{x}_{i}, t\right)$ is a smooth function, seeing $[1-3,5]$. If the ETC is designed in the channel of controller to actuator like [40-44], it has $u(t)=v\left(t_{j}\right)$ for the inter-event time $t \in\left(t_{j}, t_{j+1}\right]$, where $v(t)=h_{n}(x, t)$ is the command control signal. Denote the tracking error in the $i$ th step as $z_{i+1}=x_{i+1}-\alpha_{i}$. It can be inferred that the update of $u(t)$ at $t_{j}$ will not affect the continuity of $\alpha_{i}$ and $z_{i}$. Nevertheless, if the ETC is designed in the channel of sensor to controller, things are totally different. The definition of the problem of "jumps of virtual control laws" is provided as follows.

Definition 1 For the backstepping-based ETC in the channel of sensor to controller, the states are eventtriggered sampled in the controller, such that $\alpha_{i}(t)=$ $h_{i}\left(\bar{x}_{i}\left(t_{j}\right), t\right)$ holds in $t \in\left(t_{j}, t_{j+1}\right]$. Because $x_{i}\left(t_{j}\right) \neq$ $x_{i}\left(t_{j-1}\right)$, there is $\alpha_{i}\left(t_{j}\right)=h_{i}\left(\bar{x}_{i}\left(t_{j-1}\right), t_{j}\right)$ and $\alpha_{i}^{+}\left(t_{j}\right)=$ $h_{i}\left(\bar{x}_{i}\left(t_{j}\right), t_{j}\right)$ at $t_{j}$, such that $\Delta \alpha_{i} \neq 0$ and the jump happens. Although $x_{i}^{+}\left(t_{j}\right)=x_{i}\left(t_{j}\right), z_{i}^{+}\left(t_{j}\right) \neq z_{i}\left(t_{j}\right)$ due to $\Delta \alpha_{i} \neq 0$ and the Lyapunov function of $V\left(z_{i}\right)$ is also discontinuous at $t_{j}$.

In $[37-39,45]$, it presumed $\Delta \alpha_{i}=0$ at $t_{j}$. This disposal is not reasonable according to the above analysis. In [36], $\alpha_{i}$ was not fabricated in the NN-based controller, such that this problem was solved. In our previous work [52], this problem was solved by finding a continuous substitute for $\alpha_{i}$.

\subsection{Approximation of FLSs}

The FLSs are employed to approximate the unknown dynamics in (1). A FLS works mainly through three steps, namely fuzzification, fuzzy reasoning and defuzzification. The fuzzy rule base is consulted to conduct the entire procedure, and it is composed of the IF-THEN rules as $G^{l}$

$R^{l}$ : If $x_{1}$ is $F_{1}^{l}, x_{2}$ is $F_{2}^{l}, \cdots$ and $x_{n}$ is $F_{n}^{l}$, then $y$ is

where $l=1, \cdots, N, x=\left[x_{1}, \cdots, x_{n}\right]^{\mathrm{T}}$ is the input vector, $y$ is the output, $F_{i}^{l}$ and $G^{l}$ are the fuzzy sets with the membership functions of $\mu_{F_{i}^{l}}\left(x_{i}\right)$ and $\mu_{G^{l}}(y)$ respectively. Through the singleton fuzzification, the product reasoning and the center average defuzzification, the FLS can be rewritten as

$y=\frac{\sum_{l=1}^{N} \bar{y}_{l} \prod_{i=1}^{n} \mu_{F_{i}^{l}}\left(x_{i}\right)}{\sum_{l=1}^{N} \prod_{i=1}^{n} \mu_{F_{i}^{l}}\left(x_{i}\right)}$

where $\bar{y}_{l}=\max _{y \in \mathcal{R}} \mu_{G^{l}}(y)$. For simplicity, denote the fuzzy basis function in (2) as

$\varphi_{f l}(x)=\frac{\prod_{i=1}^{n} \mu_{F_{i}^{l}}\left(x_{i}\right)}{\sum_{l=1}^{N} \prod_{i=1}^{n} \mu_{F_{i}^{l}}\left(x_{i}\right)}$ and let $\varphi(x)=\left[\varphi_{f 1}, \cdots, \varphi_{f N}\right]^{\mathrm{T}}$ and $W=\left[\bar{y}_{1}, \cdots, \bar{y}_{N}\right]^{\mathrm{T}}$. Then, (2) can be simplified as $y=W^{\mathrm{T}} \varphi(x)$. The wellknown approximation property of the FLS is concluded as follows.

Lemma 1 For any multiple-inputs-single-output function $f(x)$ defined in a compact set $x \in \Omega$, there always exists the FLS satisfying

$f(x)=W^{\mathrm{T}} \varphi(x)+\varepsilon$

where $\varepsilon$ can be deemed as a slow time-varying variable bounded by $|\varepsilon| \leq b_{\varepsilon}$, where $b_{\varepsilon}$ is a positive constant. In this paper, $W$ is determined as the optimal weight vector satisfying $W=\arg \min _{W \in R^{N}}\left\{b_{\varepsilon}\right\}$

\section{Control Design}

The framework of the proposed control scheme is illuminated in Fig. 1. It belongs to the second configuration in [48], in which the event-triggered adaptive model and the update laws are co-located with the controller. This configuration allows for the distributed layout of sensors, and is more practical than the first configuration in [48], namely the sensor-located configuration. The control design is divided into three steps.

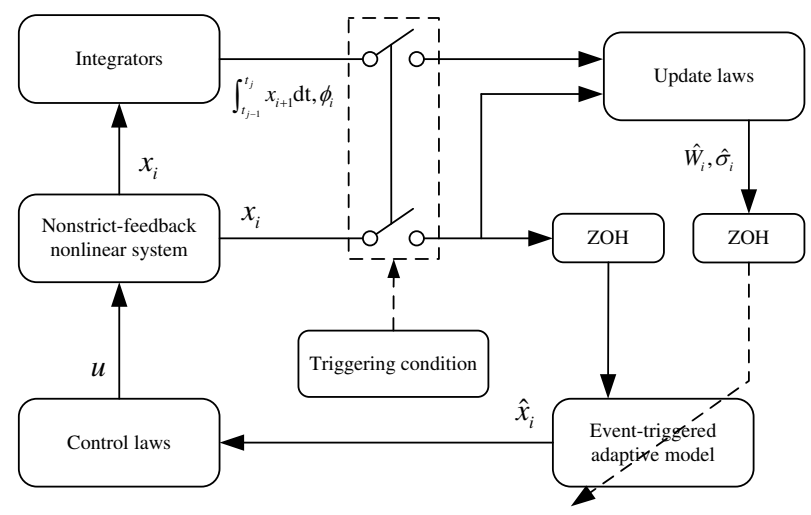

Fig. 1 Framework of proposed ETC

\subsection{Event-triggered Adaptive Model}

According to Lemma 1, the unknown dynamics in (1) is expressed as $f_{i}(x)=W_{i} \varphi_{i}(x)+\varepsilon_{i}$. According to [30], it is impractical to distinguish $\varepsilon_{i}$ from $d_{i}$. Thus, the compound disturbance is constructed with the form of 
$\sigma_{i}=\varepsilon_{i}+d_{i}$. According to (1), the event-triggered adaptive model in $t \in\left(t_{j}, t_{j+1}\right]$ is designed as

$$
\left\{\begin{array}{l}
\dot{\hat{x}}_{i}=\hat{W}_{i}^{\mathrm{T}} \varphi_{i}\left(x\left(t_{j}\right)\right)+\hat{x}_{i+1}+\hat{\sigma}_{i}+k_{i}\left(x_{i}\left(t_{j}\right)-\hat{x}_{i}\right), \\
i=1, \cdots, n-1 \\
\dot{\hat{x}}_{n}=\hat{W}_{n}^{\mathrm{T}} \varphi_{n}\left(x\left(t_{j}\right)\right)+u+\hat{\sigma}_{n}+k_{n}\left(x_{n}\left(t_{j}\right)-\hat{x}_{n}\right)
\end{array}\right.
$$

where $k_{i}$ and $k_{n}$ are tuning parameters to be designed later. It is observed that $x\left(t_{j}\right)$ in the right side of (4) is renewed at the triggering instant and $\hat{x}_{i}$ keeps continuous. Denote the measurement error as $e_{i}=x_{i}\left(t_{j}\right)-x_{i}$ and $e_{n}=x_{n}\left(t_{j}\right)-x_{n}$. By subtracting (4) from (1), it renders the estimation error system in $t \in\left(t_{j}, t_{j+1}\right]$ as

$$
\left\{\begin{aligned}
\dot{\tilde{x}}_{i}= & \tilde{W}_{i}^{\mathrm{T}} \varphi_{i}(x)+\hat{W}_{i}^{\mathrm{T}}\left(\varphi_{i}(x)-\varphi_{i}\left(x\left(t_{j}\right)\right)\right)+\tilde{x}_{i+1} \\
& +\tilde{\sigma}_{i}-k_{i} e_{i}-k_{i} \tilde{x}_{i}, i=1, \cdots, n-1 \\
\dot{\tilde{x}}_{n}= & \tilde{W}_{n}^{\mathrm{T}} \varphi_{n}(x)+\hat{W}_{n}^{\mathrm{T}}\left(\varphi_{n}(x)-\varphi_{n}\left(x\left(t_{j}\right)\right)\right)+\tilde{\sigma}_{n} \\
& -k_{n} e_{n}-k_{n} \tilde{x}_{n}
\end{aligned}\right.
$$

It can be inferred from (5) that if the inter-event time is small enough (namely $k_{i} e_{i} \rightarrow 0$ and $\hat{W}_{i}^{\mathrm{T}}\left(\varphi_{i}(x)-\right.$ $\left.\varphi_{i}\left(x\left(t_{j}\right)\right)\right) \rightarrow 0$ ) and the identification is accurate enough (namely $\tilde{W}_{i}^{\mathrm{T}} \varphi_{i}(x) \rightarrow 0$ and $\tilde{\sigma}_{i} \rightarrow 0$ ), the fast convergence of $\hat{x}_{i}$ to $x_{i}$ is ensured.

\subsection{Event-triggered Compound learning}

The update laws of $\hat{W}_{i}$ and $\hat{\sigma}_{i}$ will be fabricated to guarantee the satisfactory learning performance of $\hat{W}_{i}^{\mathrm{T}} \varphi(x)$ and $\hat{\sigma}_{i}$. According to (5), the prediction error is constructed as

$$
\left\{\begin{aligned}
s_{i}\left(t_{j}\right)= & \int_{t_{j-1}}^{t_{j}} \dot{\tilde{x}}_{i}-\hat{W}_{i}^{\mathrm{T}}\left(\varphi_{i}-\varphi_{i}\left(t_{j-1}\right)\right)-\tilde{x}_{i+1}+\hat{\sigma}_{i} \\
& +k_{i}\left(x_{i}\left(t_{j-1}\right)-\hat{x}_{i}\right) \mathrm{d} t, i=1, \cdots, n-1 \\
s_{n}\left(t_{j}\right)= & \int_{t_{j-1}}^{t_{j}} \dot{\tilde{x}}_{n}-\hat{W}_{n}^{\mathrm{T}}\left(\varphi_{n}-\varphi_{n}\left(t_{j-1}\right)\right)+\hat{\sigma}_{n} \\
& +k_{n}\left(x_{n}\left(t_{j-1}\right)-\hat{x}_{n}\right) \mathrm{d} t
\end{aligned}\right.
$$

It is clear in (6) that the prediction errors $s_{i}$ and $s_{n}$ utilize the recorded data during the previous inter-event time. As $\hat{W}_{i}$ and $\hat{\sigma}_{i}$ are only updated at the triggering instant, (6) can be further transformed to

$$
\left\{\begin{aligned}
s_{i}\left(t_{j}\right)= & \tilde{x}_{i}\left(t_{j}\right)-\tilde{x}_{i}\left(t_{j-1}\right)-\hat{W}_{i}^{+}\left(t_{j-1}\right)^{\mathrm{T}} \phi_{i}\left(t_{j}\right) \\
& +\hat{W}_{i}^{+}\left(t_{j-1}\right)^{\mathrm{T}} \varphi_{i}\left(t_{j-1}\right)\left(t_{j}-t_{j-1}\right) \\
& -\int_{t_{j-1}}^{t_{j}} x_{i+1} \mathrm{~d} t+\int_{t_{j-1}}^{t_{j}} \hat{x}_{i+1} \mathrm{~d} t \\
& +\hat{\sigma}_{i}^{+}\left(t_{j-1}\right)\left(t_{j}-t_{j-1}\right)+k_{i} x_{i}\left(t_{j-1}\right)\left(t_{j}-t_{j-1}\right) \\
& -k_{i} \int_{t_{j-1}}^{t_{j}} \hat{x}_{i} \mathrm{~d} t, i=1, \cdots, n-1 \\
s_{n}\left(t_{j}\right)= & \tilde{x}_{n}\left(t_{j}\right)-\tilde{x}_{n}\left(t_{j-1}\right)-\hat{W}_{n}^{+}\left(t_{j-1}\right)^{\mathrm{T}} \phi_{n}\left(t_{j}\right) \\
& +\hat{W}_{n}^{+}\left(t_{j-1}\right)^{\mathrm{T}} \varphi_{n}\left(t_{j-1}\right)\left(t_{j}-t_{j-1}\right) \\
& +\hat{\sigma}_{n}^{+}\left(t_{j-1}\right)\left(t_{j}-t_{j-1}\right)-k_{n} \int_{t_{j-1}}^{t_{j}} \hat{x}_{n} \mathrm{~d} t \\
& +k_{n} x_{n}\left(t_{j-1}\right)\left(t_{j}-t_{j-1}\right)
\end{aligned}\right.
$$

where $\phi_{i}\left(t_{j}\right)=\int_{t_{j-1}}^{t_{j}} \varphi_{i}(t) \mathrm{d} t$ and $\phi_{n}\left(t_{j}\right)=\int_{t_{j-1}}^{t_{j}} \varphi_{n}(t) \mathrm{d} t$. It can be inferred from (7) that the recorded data are only transmitted to the controller at the triggering instant through the integrators in Fig. 1. By substituting (5) to (6), it further renders

$$
\begin{aligned}
s_{i}\left(t_{j}\right) & =\int_{t_{j-1}}^{t_{j}} \tilde{W}_{i}^{\mathrm{T}} \varphi_{i}(x)+\sigma_{i} \mathrm{~d} t \\
& =\tilde{W}_{i}^{\mathrm{T}} \phi_{i}\left(t_{j}\right)+D_{i}\left(t_{j}\right), i=1, \cdots, n
\end{aligned}
$$

where $D_{i}\left(t_{j}\right)=\int_{t_{j-1}}^{t_{j}} \sigma_{i} \mathrm{~d} t$. According to (8), the update laws of $\hat{W}_{i}$ are designed as

$\left\{\begin{array}{l}\hat{W}_{i}^{+}\left(t_{j}\right)=l_{w i} \hat{W}_{i}\left(t_{j}\right)+\gamma_{w i} s_{i}\left(t_{j}\right) \phi_{i}\left(t_{j}\right), t=t_{j} \\ \hat{W}_{i}(t)=\hat{W}_{i}^{+}\left(t_{j}\right), t \in\left(t_{j}, t_{j+1}\right]\end{array}\right.$

where $\gamma_{w i}>0$ denotes the learning rate, $l_{w i} \hat{W}_{i}\left(t_{j}\right)$ with $l_{w i}>0$ is used to prevent the parameter drift. The selection principle of $\gamma_{w i}$ and $l_{w i}$ is discussed in Appendix A.

According to (1), the prediction error is designed as

$$
\left\{\begin{array}{l}
p_{i}\left(t_{j}\right)=\int_{t_{j-1}}^{t_{j}} \dot{x}_{i}-\hat{W}_{i}^{\mathrm{T}} \varphi_{i}(x)-x_{i+1}-\hat{\sigma}_{i} \mathrm{~d} t \\
i=1, \cdots, n-1 \\
p_{n}\left(t_{j}\right)=\int_{t_{j-1}}^{t_{j}} \dot{x}_{n}-\hat{W}_{n}^{\mathrm{T}} \varphi_{n}(x)-u-\hat{\sigma}_{n} \mathrm{~d} t
\end{array}\right.
$$


Similar with (7), it can be transformed to

$$
\left\{\begin{aligned}
p_{i}\left(t_{j}\right)= & x_{i}\left(t_{j}\right)-x_{i}\left(t_{j-1}\right)-\hat{W}_{i}^{+}\left(t_{j-1}\right)^{\mathrm{T}} \phi_{i}\left(t_{j}\right) \\
& -\int_{t_{j-1}}^{t_{j}} x_{i+1} \mathrm{~d} t-\hat{\sigma}_{i}^{+}\left(t_{j-1}\right)\left(t_{j}-t_{j-1}\right), \\
& i=1, \cdots, n-1 \\
p_{n}\left(t_{j}\right)= & x_{n}\left(t_{j}\right)-x_{n}\left(t_{j-1}\right)-\hat{W}_{n}^{+}\left(t_{j-1}\right)^{\mathrm{T}} \phi_{n}\left(t_{j}\right) \\
& -\int_{t_{j-1}}^{t_{j}} u \mathrm{~d} t-\hat{\sigma}_{n}^{+}\left(t_{j-1}\right)\left(t_{j}-t_{j-1}\right)
\end{aligned}\right.
$$

(11) illustrates that all the data are achievable for $p_{i}$ and $p_{n}$ at the triggering instant. By substituting (1) to (10), it renders

$$
\begin{aligned}
p_{i}\left(t_{j}\right) & =\int_{t_{j-1}}^{t_{j}} \tilde{W}_{i}^{\mathrm{T}} \varphi_{i}(x)+\tilde{\sigma}_{i} \mathrm{~d} t \\
& =\tilde{W}_{i}^{\mathrm{T}} \phi_{i}\left(t_{j}\right)+D_{i}\left(t_{j}\right)-\hat{\sigma}_{i}\left(t_{j}-t_{j-1}\right), i=1, \cdots, n
\end{aligned}
$$

According to (12), the update laws of $\hat{\sigma}_{i}$ are designed as

$$
\left\{\begin{array}{l}
\hat{\sigma}_{i}^{+}\left(t_{j}\right)=\left(l_{\sigma i}+\gamma_{\sigma i}\left(t_{j}-t_{j-1}\right)\right) \hat{\sigma}_{i}\left(t_{i}\right)+\gamma_{\sigma i} p_{i}\left(t_{j}\right), t=t_{j} \\
\hat{\sigma}_{i}(t)=\hat{\sigma}_{i}^{+}\left(t_{j}\right), t \in\left(t_{j}, t_{j+1}\right]
\end{array}\right.
$$

where $\gamma_{\sigma i}>0$ and $l_{\sigma i}>0$ have the same definitions with those in (9). The proposed update laws of (9) and (12) can guarantee the boundedness of $\left\|\tilde{W}_{i}\right\|$ and $\left|\tilde{\sigma}_{i}\right|$ all along the control process. The proof is provided in Appendix A.

\subsection{Control Laws}

The control laws herein are designed by referring to the event-triggered adaptive model (4) instead of the original system (1). Denote the tracking errors as $z_{1}=$ $\hat{x}_{1}-y_{d}$ and $z_{i}=\hat{x}_{i}-\beta_{i-1}$ with $i=1, \cdots, n$, where $\beta_{i}$ is the filtered variable of the virtual control law $\alpha_{i}$ and $q_{i}=\beta_{i}-\alpha_{i}$. The control design is divided into three parts.

Step 1: According to (4), the backstepping method is employed to eliminate the tracking error of $z_{1}$, and the first virtual control law in $t \in\left(t_{j}, t_{j+1}\right]$ is designed as

$$
\alpha_{1}=-k_{a 1} z_{1}-\hat{W}_{1}^{\mathrm{T}} \varphi_{1}\left(x\left(t_{j}\right)\right)-\hat{\sigma}_{1}+\dot{y}_{d}
$$

where $k_{a 1}$ is the positive tuning parameter to be discussed later. Differentiating $z_{1}$ along (4) and (14), it renders

$$
\dot{z}_{1}=-k_{a 1} z_{1}+z_{2}+q_{1}+k_{1} e_{1}+k_{1} \tilde{x}_{1}
$$

Step $i$ : Similarly to eliminate $z_{i}$ with $i=2, \cdots, n-$ 1 , the virtual control law in the $i$ th step is designed as $\alpha_{i}=-k_{a i} z_{i}-z_{i-1}-\hat{W}_{i}^{\mathrm{T}} \varphi_{i}\left(x\left(t_{j}\right)\right)-\hat{\sigma}_{i}+\dot{\beta}_{i-1}$

where $k_{a 2}$ is the positive tuning parameter to be discussed later. $\beta_{i}$ and $\dot{\beta}_{i}$ with $i=1, \cdots, n-1$ are generated by the following second-order command filter [54].

$\left\{\begin{array}{l}\dot{\beta}_{i}=\eta_{i} \\ \dot{\eta}_{i}=-2 \zeta \omega \eta_{i}-\omega^{2}\left(\beta_{i}-\alpha_{i}\right)\end{array}\right.$

where $\zeta>0$ is the damping coefficient and $\omega>0$ is the natural frequency, and it has $\dot{\beta}_{i}=\eta_{i}$. According to the definition of $q_{i},(16)$ and (17), $\Delta q_{i}$ at $t_{j}$ is expressed as

$$
\begin{aligned}
\Delta q_{i}= & \beta_{i}^{+}\left(t_{j}\right)-\alpha_{i}^{+}\left(t_{j}\right)-\beta_{i}\left(t_{j}\right)+\alpha_{i}\left(t_{j}\right) \\
= & \alpha_{i}\left(t_{j}\right)-\alpha_{i}^{+}\left(t_{j}\right) \\
= & \hat{W}_{i}^{+}\left(t_{j}\right)^{\mathrm{T}} \varphi_{i}\left(t_{j}\right)-\hat{W}_{i}^{\mathrm{T}}\left(t_{j}\right) \varphi_{i}\left(t_{j-1}\right)+\hat{\sigma}_{i}^{+}\left(t_{j}\right) \\
& -\hat{\sigma}_{i}\left(t_{j}\right)
\end{aligned}
$$

By invoking (3), the following relationship holds

$$
\begin{aligned}
\hat{W}_{i}^{\mathrm{T}} \varphi_{i} & =\sqrt{\hat{W}_{i}^{\mathrm{T}} \varphi_{i} \varphi_{i}^{\mathrm{T}} \hat{W}_{i}} \leq \sqrt{\lambda_{\max }\left(\varphi_{i} \varphi_{i}^{\mathrm{T}}\right)\left\|\hat{W}_{i}\right\|^{2}} \\
& \leq\left\|\hat{W}_{i}\right\| \leq\left\|\tilde{W}_{i}\right\|+\left\|W_{i}\right\|
\end{aligned}
$$

By invoking Assumption 1 and Lemma 1, (18) can be further expressed as

$$
\begin{aligned}
\Delta q_{i} & \leq 2\left\|\tilde{W}_{i}\right\|+2\left\|W_{i}\right\|+2\left|\tilde{\sigma}_{i}\right|+2\left|\sigma_{i}\right| \\
& \leq 2\left\|\tilde{W}_{i}\right\|+2\left\|W_{i}\right\|+2\left|\tilde{\sigma}_{i}\right|+2 b_{\varepsilon_{i}}+2 b_{d_{i}}
\end{aligned}
$$

As is proved in Appendix A that $\left\|\tilde{W}_{i}\right\|$ and $\left|\tilde{\sigma}_{i}\right|$ are bounded all along, it is reasonable that to assume a positive constant $b_{q_{i}}$ such that $\left|\Delta q_{i}\right| \leq b_{q_{i}}$ always holds. Then, it is proved in Appendix B that (17) has the following property.

Lemma 2 There exists a positive constant $\bar{b}_{q_{i}}$ such that $\left|q_{i}\right| \leq \bar{b}_{q_{i}}$ always holds. By increasing $\omega$ in (17), it has $\bar{b}_{q_{i}} \rightarrow b_{q_{i}}$

Remark 2 Because $\hat{x}_{i}$ is continuous in (4) and $\alpha_{i}$ is filtered by (17), the components of $-k_{a i} z_{i}$ and $-z_{i-1}$ in (14) and (16) are continuous at $t_{j}$. Different with the first-order DSC filter in [52], the second-order command filter of (17) can also guarantee the continuity of $\dot{\beta}_{i-1}$ in (16), such that the sophisticated analysis of $\Delta \dot{\beta}_{i}$ is avoided.

Differentiating $z_{i}$ along (4) and (16), it renders

$\dot{z}_{i}=-k_{a i} z_{i}+z_{i+1}+q_{i}-z_{i-1}+k_{i} e_{i}+k_{i} \tilde{x}_{i}$ 
Step $n$ : Similarly to eliminate $z_{n}$, the real control law of $u$ in the final step is designed as

$u=-k_{a n} z_{n}-z_{n-1}-\hat{W}_{n}^{\mathrm{T}} \varphi_{n}\left(x\left(t_{j}\right)\right)-\hat{\sigma}_{n}+\dot{\beta}_{n-1}$

By differentiating $z_{n}$ along (4) and (16), it renders

$\dot{z}_{n}=-k_{a n} z_{n}-z_{n-1}+k_{n} e_{n}+k_{n} \tilde{x}_{n}$

Remark 3 Following from (1), the essence of "algebraic loop" problem implies that the $x_{i}$ can not converge to the $\alpha_{i-1}(x)$ in the backstepping method which is synchronously changed. Whereas in the event-triggered adaptive model (4), the convergence of $\hat{x}_{i}$ to $\beta_{i-1}$ will not cause the synchronous change of $\alpha_{i-1}\left(x, \hat{x}_{i-1}\right)$. Here, $\hat{x}_{i}$ can only affect the derivative of $x$, which is same as $x_{i}$ can only affect the derivative of $x_{i-1}$ in the strictfeedback system. Moreover in the proposed scheme, $x$ is not continuously sampled in $\alpha_{i-1}$. Thus, the "algebraic loop" problem will never occur.

\section{Triggering Condition and Stability Analysis}

\subsection{Design of Triggering Condition}

Select the Lyapunov function as $V_{1}=\sum_{i=1}^{n} \tilde{x}_{i}^{2} / 2$. Differentiating $V_{1}$ along (5), it renders

$$
\begin{aligned}
\dot{V}_{1} \leq & -\left(k_{1}-\frac{5}{2}\right) \tilde{x}_{1}^{2}-\sum_{i=2}^{n-1}\left(k_{i}-3\right) \tilde{x}_{i}^{2}-\left(k_{n}-\frac{5}{2}\right) \tilde{x}_{n}^{2} \\
& +\sum_{i=1}^{n}\left(\frac{1}{2} \hat{W}_{i}^{\mathrm{T}}\left(\varphi_{i}-\varphi_{i}\left(t_{j}\right)\right)\left(\varphi_{i}^{\mathrm{T}}-\varphi_{i}^{\mathrm{T}}\left(t_{j}\right)\right) \hat{W}_{i}+\frac{1}{2} k_{i}^{2} e_{i}^{2}\right) \\
& +\sum_{i=1}^{n}\left(\frac{1}{2}\left\|\tilde{W}_{i}\right\|^{2}+\frac{1}{2} \tilde{\sigma}_{i}^{2}\right)
\end{aligned}
$$

Select the Lyapunov function as $V_{2}=\sum_{i=1}^{n} z_{i}^{2} / 2$. Differentiating $V_{2}$ along (15), (21) and (23), it renders

$$
\begin{aligned}
\dot{V}_{2} & =-\sum_{i=1}^{n} k_{a i} z_{i}^{2}+\sum_{i=1}^{n-1} q_{i} z_{i}+\sum_{i=1}^{n}\left(k_{i} e_{i} z_{i}+k_{i} \tilde{x}_{i} z_{i}\right) \\
& \leq-\sum_{i=1}^{n}\left(k_{a i}-1-\frac{k_{i}^{2}}{2}\right) z_{i}^{2}+\sum_{i=1}^{n-1} \frac{q_{i}^{2}}{2}+\sum_{i=1}^{n}\left(\frac{k_{i}^{2} e_{i}^{2}}{2}+\frac{\tilde{x}_{i}^{2}}{2}\right)
\end{aligned}
$$

Define $V=V_{1}+V_{2}$. By adding up (24) and (25) and invoking Lemma 2, the differential of $V$ is obtained as

$$
\begin{aligned}
\dot{V} \leq & -\left(k_{1}-3\right) \tilde{x}_{1}^{2}-\sum_{i=2}^{n-1}\left(k_{i}-\frac{7}{2}\right) \tilde{x}_{i}^{2}-\left(k_{n}-3\right) \tilde{x}_{n}^{2} \\
& -\sum_{i=1}^{n}\left(k_{a i}-1-\frac{k_{i}^{2}}{2}\right) z_{i}^{2}+\sum_{i=1}^{n-1} \frac{\bar{b}_{q_{i}}^{2}}{2}+\sum_{i=1}^{n} b_{l_{i}} \\
& +\sum_{i=1}^{n}\left(\frac{1}{2} \hat{W}_{i}^{\mathrm{T}}\left(\varphi_{i}-\varphi_{i}\left(t_{j}\right)\right)\left(\varphi_{i}^{\mathrm{T}}-\varphi_{i}^{\mathrm{T}}\left(t_{j}\right)\right) \hat{W}_{i}+k_{i}^{2} e_{i}^{2}\right)
\end{aligned}
$$

where $b_{l_{i}}=\sup \left\{\left\|\tilde{W}_{i}\right\|^{2} / 2+\tilde{\sigma}_{i}^{2} / 2\right\}$. Because $\left\|\tilde{W}_{i}\right\|$ and $\left|\tilde{\sigma}_{i}\right|$ are bounded all along according to the proof in Appendix A, $b_{l_{i}}$ is an unknown constant.

According to (26), the triggering condition is designed as

$\sum_{i=1}^{n}\left(\frac{1}{2} \hat{W}_{i}^{\mathrm{T}}\left(\varphi_{i}-\varphi_{i}\left(t_{j}\right)\right)\left(\varphi_{i}^{\mathrm{T}}-\varphi_{i}^{\mathrm{T}}\left(t_{j}\right)\right) \hat{W}_{i}+k_{i}^{2} e_{i}^{2}\right)<\mu$

where $\mu$ is the positive triggering threshold. The next triggering instant is activated while (27) is violated. The proof of the existence of minimum inter-event time $\delta_{t}$ (namely to avoid the "Zeno" behavior) adopts the same thought in [49-51], which is briefly introduced as follows.

Proof of $\delta_{t}$ : It is observed that the left side of (27) equates to 0 at the triggering instant. Because all the states are defined in a compact set, the left side of (27) must have a limited growth rate. As $\mu$ is a positive constant, it takes at least the time of $\delta_{t}$ to increase from 0 to $\mu$.

\subsection{Stability Analysis}

The main result of this paper is concluded as the following theorem.

Theorem 1 Assume all the states of (1) are defined in a compact set, namely $\Omega_{x}=\left\{x \mid x^{\mathrm{T}} x \leq b_{\Omega_{x}}\right\}$ where $b_{\Omega_{s}}$ is a positive constant. If the update laws of (9) and (13), the real control law of (22), and the triggering condition of (27) are used, all the tracking errors are proved to satisfy SGUUB. Moreover, by tuning the parameters appropriately, the output $y$ can track the reference $y_{d}$ in an arbitrarily small precision.

Proof: By substituting (27) to (26), and selecting $a=$ $2 \min \left\{k_{1}-3, k_{i}-7 / 2, k_{n}-3, k_{a i}-1-k_{i}^{2} / 2\right\}$ and $b=$ $\sum_{i=1}^{n-1} \bar{b}_{q_{i}}^{2} / 2+\sum_{i=1}^{n} b_{l_{i}}+\mu,(26)$ can be simplified as

$\dot{V} \leq-a V+b$ 
According to the comparison theorem, it can be inferred from (28) that for $i=1, \cdots, n$

$\left|z_{i}\right| \leq \sqrt{2 V} \leq \sqrt{2 V\left(t_{0}\right) e^{-a\left(t-t_{0}\right)}+\frac{2 b}{a}\left(1-e^{-a\left(t-t_{0}\right)}\right)}$

Thus, the SGUUB of $z_{i}$ is proved. Because $y-y_{d}=$ $z_{1}+\tilde{x}_{1}$ and $\left|\tilde{x}_{i}\right| \leq \sqrt{2 V}$ too, it is inferred that $\left|y-y_{d}\right| \leq$ $2 \sqrt{2 V}$. If $a \rightarrow+\infty$ in (29), it has $2 \sqrt{2 V} \rightarrow 0$ with $t \rightarrow+\infty$. The convergence of $y$ to $y_{d}$ is proved.

The parameter selection follows two steps: 1) select $k_{1}>3, k_{i}>7 / 2, k_{n}>3$ and $\mu>0$ to ensure the acceptable estimation performance of (4);2) select $k_{a i}>1+k_{i}^{2} / 2$ to ensure the satisfactory tracking performance.

Remark 4 According to the definition of $z_{1}$, it is inferred that $z_{1} \rightarrow 0 \Rightarrow x_{1} \rightarrow y_{d}$ if $x_{i} \rightarrow \hat{x}_{i}$. Because $\tilde{x}_{i}$ is affected by the approximation errors and the measurement errors according to (5), the tracking performance is positively associated with the learning performance and decreasing the triggering threshold $\mu$ in (27). Nevertheless, decreasing $\mu$ will increase the communication load as the inter-event time is shorten.

\section{Numerical Experiment}

In this section, the proposed ETC compound learning scheme is applied to the ball and beam system in [55], and its setup is shown in Fig. 2.

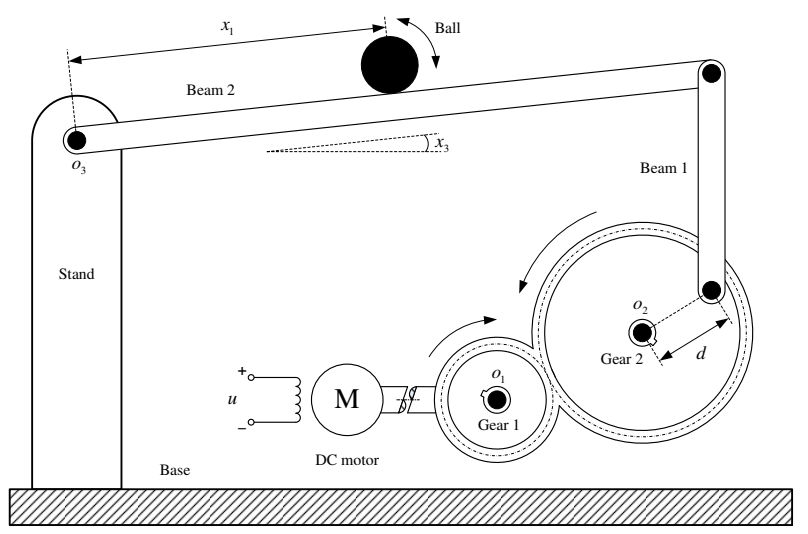

Fig. 2 Diagram of ball and beam system

In Fig. 2, $o_{1}, o_{2}$ and $o_{3}$ are three fixed pivots. By regulating the voltage of the DC motor (namely $u$ ), the position of the ball in beam 2 (namely $x_{1}$ ) can be regulated as needed. This system possesses the mathe-

\section{Table 1 MODEL PARAMETERS}

\begin{tabular}{ccc}
\hline Symbols & Definitions & Values \\
\hline$m_{B}$ & Mass of ball & $0.0217 \mathrm{~kg}$ \\
$m_{b}$ & Mass of beam & $0.334 \mathrm{~kg}$ \\
$R$ & Radius of ball & $0.00873 \mathrm{~m}$ \\
$l_{b}$ & Beam 2 length & $0.4 \mathrm{~m}$ \\
$d$ & Radius of large gear & $0.04 \mathrm{~m}$ \\
$J_{B}$ & Ball inertia & $6.803 \times 10^{-7} \mathrm{kgm}^{2}$ \\
$J_{b}$ & Beam inertia & $0.017813 \mathrm{kgm}^{2}$ \\
$K_{b}$ & Back-EMF constant & $0.1491 \mathrm{Nm} / \mathrm{A}$ \\
$R_{a}$ & Armature resistance & $18.91 \Omega$ \\
$g$ & Acceleration of gravity & $9.8 \mathrm{~m} / \mathrm{s}$ \\
$n_{g}$ & Gear ratio & 4.2 \\
\hline
\end{tabular}

matical model of

$\left\{\begin{aligned} \dot{x}_{1}= & x_{2} \\ \dot{x}_{2}= & A x_{1} x_{4}^{2}-A g x_{3}+d_{2} \\ \dot{x}_{3}= & x_{4} \\ \dot{x}_{4}= & B \cos \left(x_{3}\right)\left(C \cos \left(\frac{l_{b} x_{3}}{d}\right) u-D x_{4} \cos \left(\frac{l_{b} x_{3}}{d}\right)\right. \\ & \left.\quad-E-H x_{1}\right)-B G x_{1} x_{2} x_{4}+d_{4}\end{aligned}\right.$

where $x_{2}$ is the translational speed of the ball along beam $2, x_{3}$ is the angle of beam $2, x_{4}$ is the angular speed, $A=1 /\left(1+J_{B} /\left(m_{B} R^{2}\right)\right), B=1 /\left(J_{B}+J_{b}+\right.$ $\left.m_{B} x_{1}^{2}\right), C=n_{g} K_{b} l_{b} /\left(R_{a} d\right), D=\left(n_{g} K_{b} l_{b}\right)^{2} /\left(R_{a} d^{2}\right)$, $E=0.5 l_{b} m_{b} g, H=m_{B} g$, and $G=2 m_{B}$. The definitions and the values of the model parameters are provided in Table I. To correspond with the structure of (1), let $f_{2}(x)=A x_{1} x_{4}^{2}-A g x_{3}-x_{3}$ and $f_{4}(x)=$ $-B \cos \left(x_{3}\right)\left(D x_{4} \cos \left(l_{b} x_{3} / d\right)+E+H x_{1}\right)-B G x_{1} x_{2} x_{4}$ express the unknown dynamics in (30), and the unknown dynamics and the disturbances in the hierarchies of $x_{1}$ and $x_{3}$ are omitted. To eliminate the unknown input gain, reconstruct the control input as $U=$ $B C \cos \left(x_{3}\right) \cos \left(l_{b} x_{3} / d\right) u$.

The time-varying disturbances are described by the following second-order Markov process.

$\left\{\begin{array}{l}\ddot{d}_{2}=-\dot{d}_{2}-d_{2}+0.1 \omega_{2} \\ \ddot{d}_{4}=-\dot{d}_{4}-d_{4}+\omega_{4}\end{array}\right.$

where $\omega_{2}$ and $\omega_{4}$ are two independent Gauss white noise with the variance of 1 . The initial values of $d_{2}, \dot{d}_{2}, d_{4}$ and $\dot{d}_{4}$ are assigned as 0 . The FLS is set with 7 fuzzy rules, and the membership function for each fuzzy set is described as

$\mu_{F_{i} l}=\exp \left(-\frac{\left(x_{i}-l+4\right)^{2}}{4}\right), l=1, \cdots, 7$

It should be noted that $f_{2}(x)$ and $f_{4}(x)$ in (30) employ the same set of fuzzy membership functions in this simulation. The reference signal is selected as a sinusoidal 
function of time, namely $y_{d}=0.2+0.05 \sin (0.1257(t-$ $\left.t_{0}\right)$ ). The initial values of states are assigned as $x_{1}\left(t_{0}\right)=$ $0.3 \mathrm{~m}, x_{2}\left(t_{0}\right)=0 \mathrm{~m} / \mathrm{s}, x_{3}\left(t_{0}\right)=0^{\circ}$ and $x_{4}=0^{\circ} / \mathrm{s}$. The initial values of the estimation states in (4) and the learning parameters in (9) and (13) are assigned as zeros.

Not to deteriorate the algorithm effectiveness, the simulation adopts the constant numerical calculation period of 0.01s. To demonstrate the differences, two comparative algorithms are used in this simulation. The first one is the time-triggered compound learning, marked as "TT-CL". In this scheme, $\mu$ in (27) is set as 0. According to [30], the continuous update law of $\hat{W}_{i}$ and the disturbance observer of $\hat{\sigma}_{i}$ are presented as

$\dot{\hat{W}}_{i}=\gamma_{w i} s_{i}(t) \phi_{i}(t)-l_{w i} \hat{W}_{i}, i=1, \cdots, n$

$$
\left\{\begin{array}{l}
\hat{\sigma}_{i}=l_{\sigma i}\left(x_{i}-\delta_{i}\right) \\
\dot{\delta}_{i}=\hat{W}_{i}^{\mathrm{T}} \varphi_{i}(x)+\hat{\sigma}_{i}+x_{i+1}, i=1, \cdots, n-1 \\
\hat{\sigma}_{n}=l_{\sigma n}\left(x_{n}-\delta_{n}\right), \dot{\delta}_{n}=\hat{W}_{n}^{\mathrm{T}} \varphi_{n}(x)+\hat{\sigma}_{n}+u
\end{array}\right.
$$

where $s_{i}(t)$ in (33) is the prediction error using the data collected in the latest $0.1 \mathrm{~s}$. The second algorithm adopts the predictor-based learning in [23], which is marked as "ET-PL". In this scheme, the compound disturbance $\sigma_{i}$ is not addressed, and the update law of $\hat{W}_{i}$ is designed as

$$
\left\{\begin{array}{l}
\hat{W}_{i}^{+}\left(t_{j}\right)=l_{w i} \hat{W}_{i}\left(t_{j}\right)+\gamma_{w i} \tilde{x}_{i}\left(t_{j}\right) \varphi_{i}\left(t_{j-1}\right), t=t_{j} \\
\hat{W}_{i}(t)=\hat{W}_{i}^{+}\left(t_{j}\right), t \in\left(t_{j}, t_{j+1}\right]
\end{array}\right.
$$

where the event-triggered adaptive model (4) functions as the predictor and $\tilde{x}_{i}$ can be deemed as the prediction error.

The parameters are uniformly set as $k_{1}=k_{3}=4$, $k_{2}=k_{4}=5, \mu=0.2, k_{a 1}=k_{a 2}=k_{a 3}=k_{a 4}=15$, $l_{w 2}=l_{w 4}=l_{\sigma 2}=l_{\sigma 4}=0.1$ and $\gamma_{w 2}=\gamma_{w 4}=\gamma_{\sigma 2}=$ $\gamma_{\sigma 4}=0.1$ in the schemes. Define the total uncertainty as $\Upsilon_{i}=f_{i}(x)+d_{i}$ and its estimate as $\hat{\Upsilon}_{i}=\hat{W}_{i}^{\mathrm{T}} \varphi_{i}(x)+\hat{\sigma}_{i}$, and let $\Upsilon=\left[\Upsilon_{2}, \Upsilon_{4}\right]^{\mathrm{T}}$. To quantify the performance, four indexes are defined as

$$
\left\{\begin{array}{l}
J_{z}(\mathrm{IAE})=\int_{t_{0}}^{t}\left|y(\tau)-y_{d}(\tau)\right| \mathrm{d} \tau, J_{c}(\mathrm{IAE})=\int_{t_{0}}^{t}\|\tilde{\Upsilon}\| \mathrm{d} \tau \\
J_{z}(\mathrm{ITAE})=\int_{t_{0}}^{t} \tau\left|y-y_{d}\right| \mathrm{d} \tau, J_{c}(\mathrm{ITAE})=\int_{t_{0}}^{t} \tau\|\tilde{\Upsilon}\| \mathrm{d} \tau
\end{array}\right.
$$

The values of these indexes are provided in Table II. The simulation results are exhibited as follows.

Fig. 3 shows the evolution of disturbances. The magnitudes of them correspond with the magnitudes of

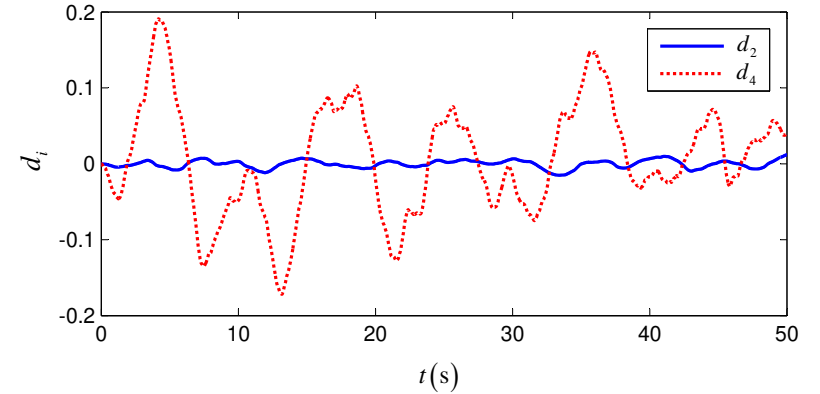

Fig. 3 Disturbance $d_{i}$

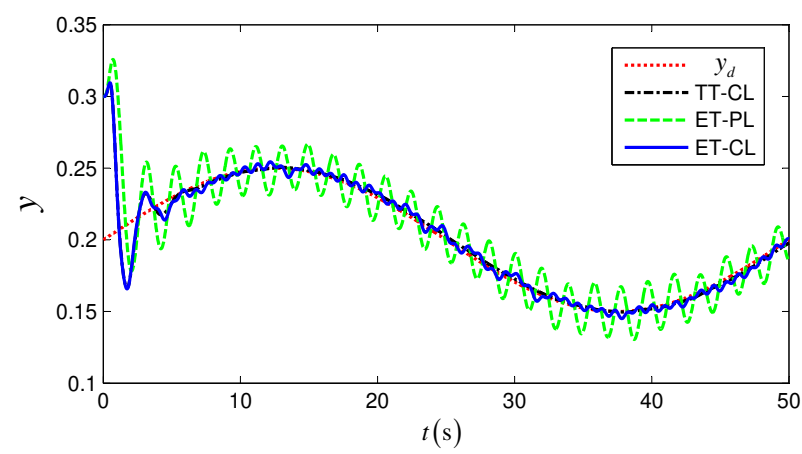

Fig. 4 Output $y$
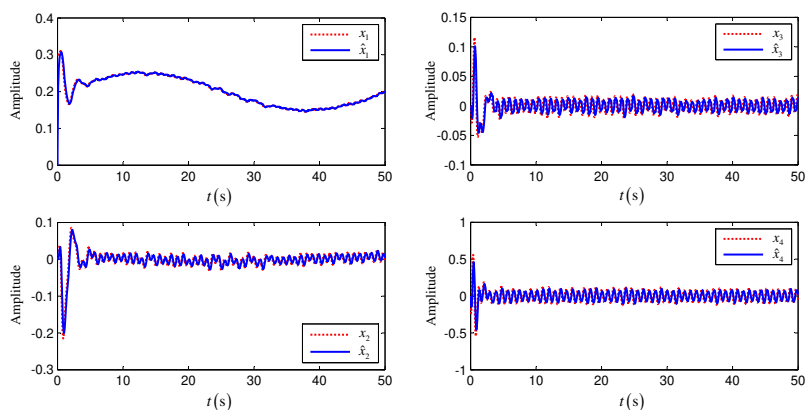

(a) $\hat{x}_{1}$ and $\hat{x}_{2}$

(b) $\hat{x}_{3}$ and $\hat{x}_{4}$

Fig. 5 States of event-triggered adaptive model in ET-CL

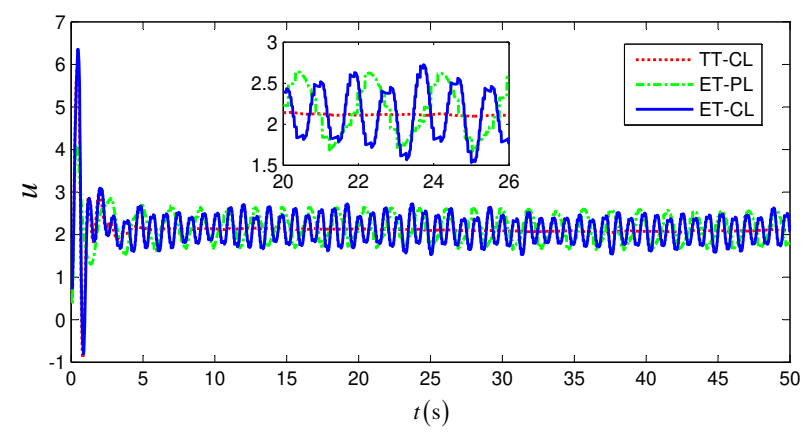

Fig. 6 Control input $u$ 
Table 2 PERFORMANCE INDEXES

\begin{tabular}{|c|c|c|c|}
\hline Indexes & TT-CL & ET-PL & ET-CL \\
\hline$J_{z}$ (IAE) & 0.215 & 0.710 & 0.254 \\
\hline$J_{z}$ (ITAE) & 2.155 & 13.699 & 3.154 \\
\hline$J_{c}$ (IAE) & 17.379 & 364.606 & 192.462 \\
\hline$J_{c}$ (ITAE) & 65.365 & 8325.970 & 4698.126 \\
\hline Total sampling times & 5001 & 593 & 1005 \\
\hline
\end{tabular}
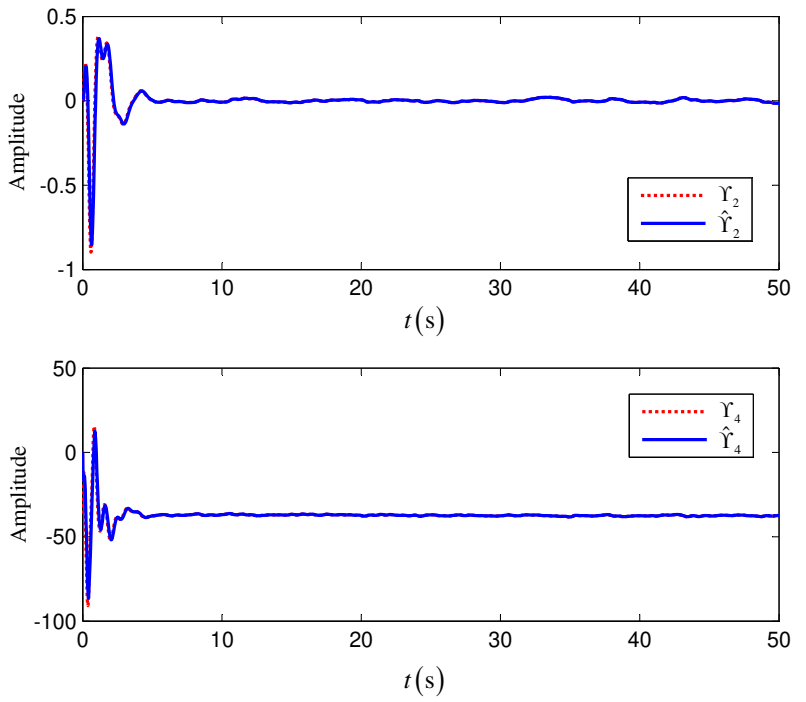

Fig. 7 Learning performance of TT-CL
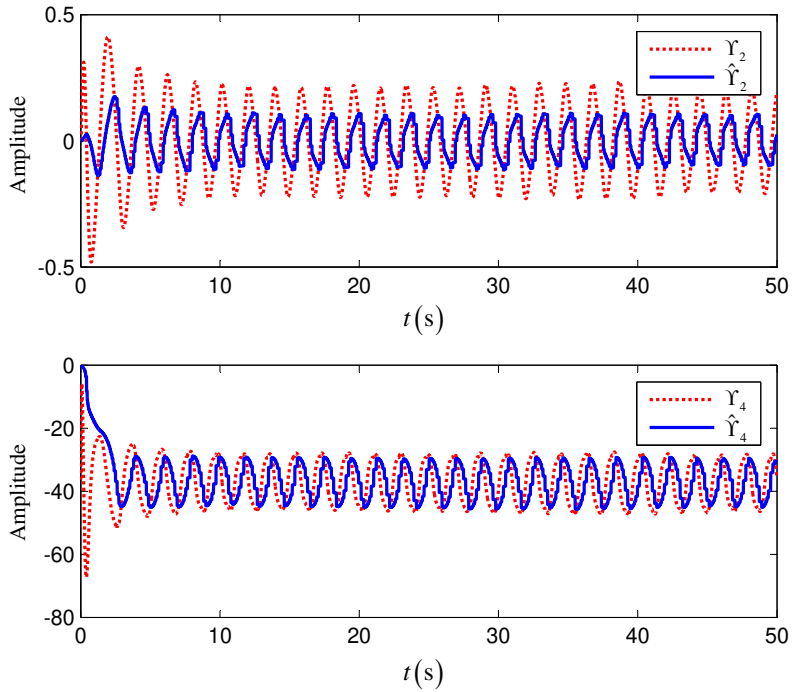

Fig. 8 Learning performance of ET-PL

total uncertainties. Fig. 4 demonstrates the output of three schemes. It is clear that TT-CL and ET-CL have better tracking performance than ET-PL due to the better learning performance of them. This point is verified in $J_{z}$ (IAE) and $J_{z}$ (ITAE) of Table II. Because the ETC is employed, the outputs of ET-CL and ET-PL inevitably suffer from fluctuation. Fig. 5 shows the evolution of $\hat{x}_{i}$ in (4) of ET-CL, in which a fast and accu-

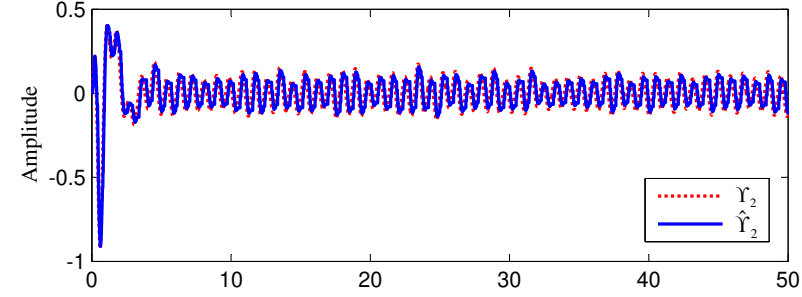

$t(\mathrm{~s})$

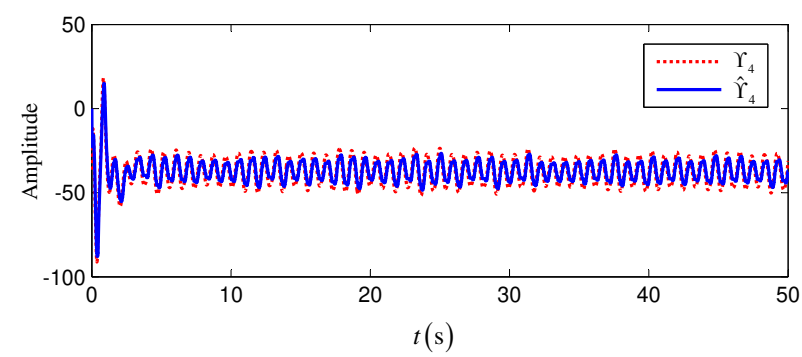

Fig. 9 Learning performance of ET-CL
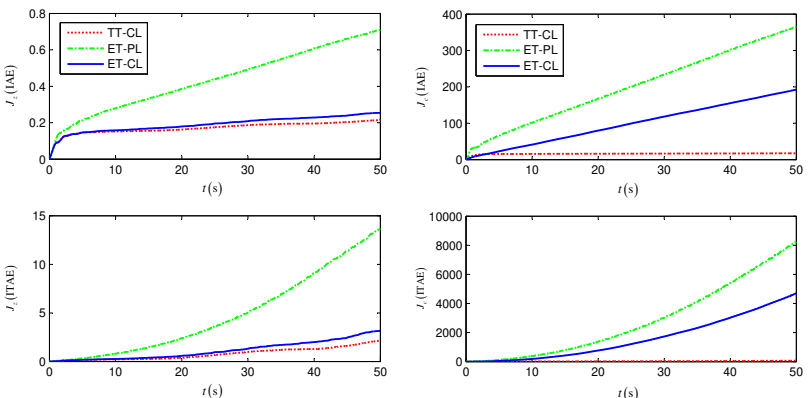

(a) $J_{z}(\mathrm{IAE})$ and $J_{z}$ (ITAE)

(b) $J_{c}(\mathrm{IAE})$ and $J_{c}$ (ITAE)

Fig. 10 Evolution of performance indexes
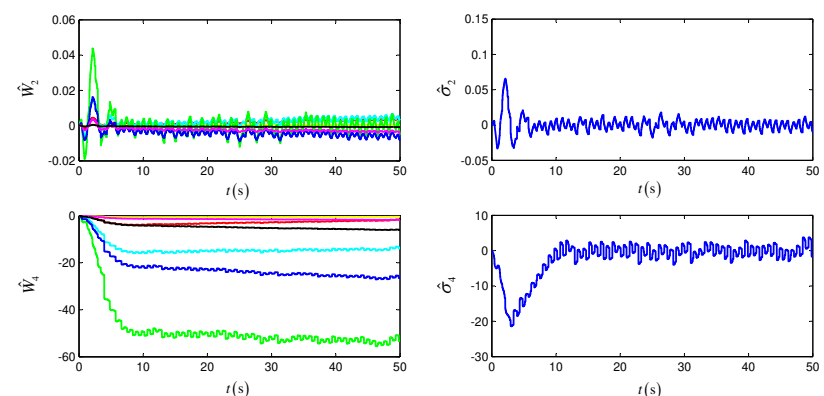

(a) Fuzzy weights $\hat{W}_{i}$

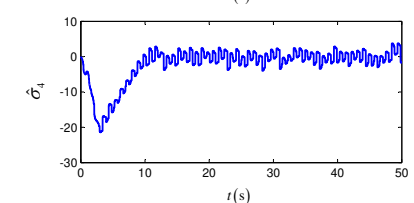

(b) Compound disturbance $\hat{\sigma}_{i}$

Fig. 11 Update laws of ET-CL

rate convergence to $x_{i}$ is observed. Fig. 6 demonstrates the control inputs of three schemes. The discontinuity of ET-CL and ET-PL is observed in the local enlarged view.

Fig. 7-9 compare the leaning performance of three schemes. Among them, TT-CL has the best learning performance, and ET-CL has much better learning performance than ET-PL. This point is verified in $J_{c}$ (IAE) 


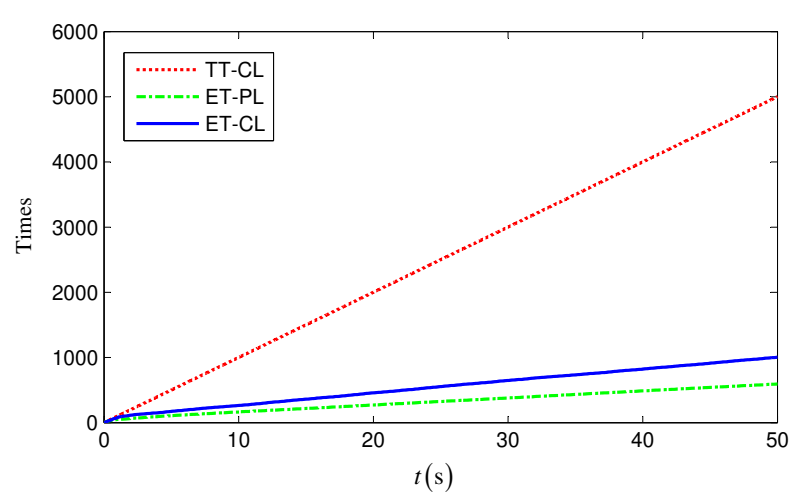

Fig. 12 Accumulation of sampling times

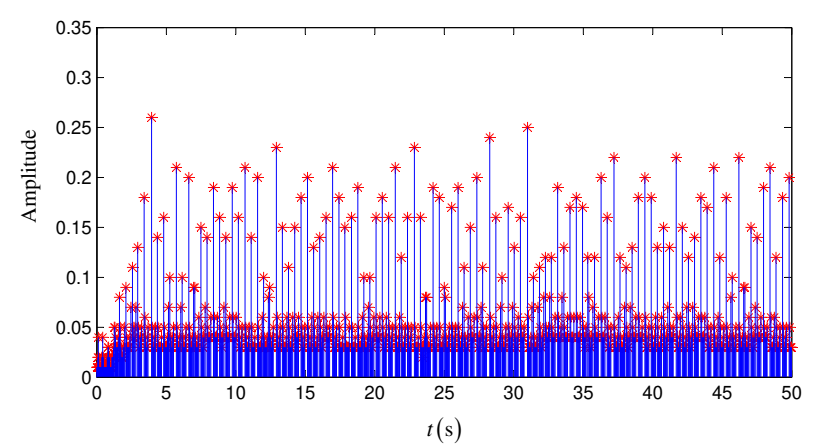

Fig. 13 Inter-event time of ET-CL

and $J_{c}$ (ITAE) of Table II. Fig. 10 shows the evolution of performance indexes. It is clear that the indexes of ETPL grow faster than the other two. Fig. 11 demonstrates the variation of update laws in ET-CL, in which the event-triggered renewal is observed. Fig. 12 compares the accumulation of sampling times in three schemes. A considerable communication saving is observed in the event-triggered schemes. The statistics of total sampling times are provided in Table II. Fig. 13 shows the evolution of inter-event time in ET-CL. The minimum inter-event time is observed at the initial stage with the constant calculation period of $0.01 \mathrm{~s}$. The maximum inter-event time is recorded as $0.26 \mathrm{~s}$.

\section{CONCLUSION}

This paper proposes an ETC scheme of the nonstrictfeedback nonlinear system with the compound learning. An event-triggered adaptive fuzzy model is fabricated to direct the backstepping design of control laws, such that the "algebraic loop" problem is solved. Two prediction errors are derived from the adaptive model and the original system, which can collect the data during the previous inter-event time. By resorting to the prediction errors, the estimates of the fuzzy weights and the compounding disturbances are updated in an event- triggered manner. In the control laws, the second-order command filters are employed to substitute for the virtual control laws and their derivatives, such that the problem of "jumps of virtual control laws" is solved. An adaptive triggering condition is constructed to guarantee the closed-loop stability. Finally, the effectiveness of the proposed scheme is exemplified in the ball and beam system via numerical simulation. In the future, we plan to incorporate the presented event-triggered compound learning technique into the nautical practice.

\section{A Proof of bounded estimation errors}

Select the Lyapunov function as $V_{w i}=\tilde{W}_{i}^{\mathrm{T}} \tilde{W}_{i}$. By invoking (8), (9) and the Young's inequality, $\Delta V_{w i}$ is derived as

$$
\begin{aligned}
\Delta V_{w i}= & -\tilde{W}_{i}^{\mathrm{T}} \tilde{W}_{i}+\left(W_{i}-l_{w i} \hat{W}_{i}-\gamma_{w i} \phi_{i} \phi_{i}^{\mathrm{T}} \tilde{W}_{i}-\gamma_{w i} \phi_{i} D_{i}\right)^{\mathrm{T}} \\
& \times\left(W_{i}-l_{w i} \hat{W}_{i}-\gamma_{w i} \phi_{i} \phi_{i}^{\mathrm{T}} \tilde{W}_{i}-\gamma_{w i} \phi_{i} D_{i}\right) \\
= & \left(l_{w i} \tilde{W}_{i}^{\mathrm{T}}+\left(1-l_{w i}\right) W_{i}^{\mathrm{T}}-\gamma_{w i} \tilde{W}_{i}^{\mathrm{T}} \phi_{i} \phi_{i}^{\mathrm{T}}-\gamma_{w i} D_{i} \phi_{i}^{\mathrm{T}}\right) \\
& \times\left(l_{w i} \tilde{W}_{i}+\left(1-l_{w i}\right) W_{i}^{\mathrm{T}}-\gamma_{w i} \phi_{i} \phi_{i}^{\mathrm{T}} \tilde{W}_{i}-\gamma_{w i} \phi_{i} D_{i}\right) \\
& -\tilde{W}_{i}^{\mathrm{T}} \tilde{W}_{i} \\
\leq & 4\left(1-l_{w i}\right)^{2} W_{i}^{\mathrm{T}} W_{i}+3 l_{w i}^{2} \tilde{W}_{i}^{\mathrm{T}} \tilde{W}_{i}+4 \gamma_{w i}^{2} D_{i}^{2} \phi_{i}^{\mathrm{T}} \phi_{i} \\
& +3 \gamma_{w i}^{2} \tilde{W}_{i}^{\mathrm{T}} \phi_{i} \phi_{i}^{\mathrm{T}} \phi_{i} \phi_{i}^{\mathrm{T}} \tilde{W}_{i}-\tilde{W}_{i}^{\mathrm{T}} \tilde{W}_{i} \\
\leq & -\left(1-3 l_{w i}^{2}-3 \gamma_{w i}^{2} n_{\phi_{i}}^{2}\right) \tilde{W}_{i}^{\mathrm{T}} \tilde{W}_{i}+4 \gamma_{w i}^{2} n_{\phi_{i}} b_{D_{i}} \\
& +4\left(1-l_{w i}\right)^{2} W_{i}^{\mathrm{T}} W_{i}
\end{aligned}
$$

where $\left\|\phi_{i}\right\|^{2} \leq n_{\phi_{i}}, D_{i}^{2} \leq b_{D_{i}}$ and $\lambda_{\max }\left(\phi_{i} \phi_{i}^{\mathrm{T}} \phi_{i} \phi_{i}^{\mathrm{T}}\right) \leq n_{\phi_{i}}^{2}$. Because the maximum inter-event time $T_{\max }$ is finite and $\left\|\varphi_{i}\right\|$ and $\sigma_{i}$ are bounded, it is tenable that the positive constants of $n_{\phi_{i}}$ and $b_{D_{i}}$ exist.

Select the Lyapunov function as $V_{\sigma i}=\tilde{\sigma}_{i}^{2}$. By invoking (12), (13) and the Young's inequality, $\Delta V_{\sigma i}$ is derived as

$$
\begin{aligned}
\Delta V_{\sigma_{i}}= & \left(\sigma_{i}-l_{\sigma i} \hat{\sigma}_{i}-\gamma_{\sigma i} \tilde{W}_{i}^{\mathrm{T}} \phi_{i}-\gamma_{\sigma i} D_{i}\right)^{2}-\tilde{\sigma}_{i}^{2} \\
= & \left(l_{\sigma i} \tilde{\sigma}_{i}+\left(1-l_{\sigma i}\right) \sigma_{i}-\gamma_{\sigma i} \tilde{W}_{i}^{\mathrm{T}} \phi_{i}-\gamma_{\sigma i} D_{i}\right)^{2}-\tilde{\sigma}_{i}^{2} \\
\leq & 4\left(1-l_{\sigma i}\right)^{2} \sigma_{i}^{2}+4 l_{\sigma i}^{2} \tilde{\sigma}_{i}^{2}+4 \gamma_{\sigma i}^{2} \tilde{W}_{i}^{\mathrm{T}} \phi_{i} \phi_{i}^{\mathrm{T}} \tilde{W}_{i}-\tilde{\sigma}_{i}^{2} \\
& +4 \gamma_{\sigma i}^{2} D_{i}^{2} \\
\leq & -\left(1-4 l_{\sigma i}^{2}\right) \tilde{\sigma}_{i}^{2}+4\left(1-l_{\sigma i}\right)^{2} b_{\sigma_{i}}+4 \gamma_{\sigma i}^{2} b_{D_{i}} \\
& +4 \gamma_{\sigma i}^{2} n_{\phi_{i}} \tilde{W}_{i}^{\mathrm{T}} \tilde{W}_{i}
\end{aligned}
$$

where $b_{\sigma_{i}}=\left(b_{d_{i}}+b_{\varepsilon_{i}}\right)^{2}$. Define $V_{i}=V_{w i}+V_{\sigma i}$. By adding up (A-1) and (A-2), it renders

$$
\begin{aligned}
\Delta V_{i} \leq & -\left(1-3 l_{w i}^{2}-3 \gamma_{w i}^{2} n_{\phi_{i}}^{2}-4 \gamma_{\sigma i}^{2} n_{\phi_{i}}\right) \tilde{W}_{i}^{\mathrm{T}} \tilde{W}_{i}+4 \gamma_{\sigma i}^{2} b_{D_{i}} \\
& -\left(1-4 l_{\sigma i}^{2}\right) \tilde{\sigma}_{i}^{2}+4 \gamma_{w i}^{2} n_{\phi_{i}} b_{D_{i}}+4\left(1-l_{w i}\right)^{2} W_{i}^{\mathrm{T}} W_{i} \\
& +4\left(1-l_{\sigma i}\right)^{2} b_{\sigma_{i}}
\end{aligned}
$$

By selecting $l_{\sigma i}<1 / 2,3 \gamma_{w i}^{2} n_{\phi_{i}}^{2}+4 \gamma_{\sigma i}^{2} n_{\phi_{i}}<1$ and $l_{w i}<$ $\left(1-3 \gamma_{w i}^{2} n_{\phi_{i}}^{2}-4 \gamma_{\sigma i}^{2} n_{\phi_{i}}\right) / \sqrt{3}$, and letting $a_{i}=\min \left\{1-3 l_{w i}^{2}-\right.$ $\left.3 \gamma_{w i}^{2} n_{\phi_{i}}^{2}-4 \gamma_{\sigma i}^{2} n_{\phi_{i}}, 1-4 l_{\sigma i}^{2}\right\}$ and $b_{i}=4 \gamma_{w i}^{2} n_{\phi_{i}} b_{D_{i}}+4(1-$ $\left.l_{w i}\right)^{2} W_{i}^{\mathrm{T}} W_{i}+4\left(1-l_{\sigma i}\right)^{2} b_{\sigma_{i}}+4 \gamma_{\sigma i}^{2} b_{D_{i}}$, (A-3) can be simplified 
as $\Delta V_{i} \leq-a_{i} V_{i}+b_{i}$. Because $\tilde{\sigma}_{i}$ is changing during the interevent time, one can not infer the boundedness of $\left|\tilde{\sigma}_{i}\right|$ from $\Delta V_{i} \leq-a_{i} V_{i}+b_{i}$. A further processing is needed.

Because $\dot{\tilde{W}}_{i}=0$ in $t \in\left(t_{j}, t_{j+1}\right]$, it is obvious that $\Theta V_{i}=\Delta V_{i}+\tilde{\sigma}_{i}^{2}\left(t_{j+1}\right)-\tilde{\sigma}_{i}^{+}\left(t_{j}\right)^{2}$. Assume $\left|\tilde{\sigma}_{i}\right|$ grows with the fastest speed during the inter-vent time. According to Assumption 1 and (13), it is tenable to assume $\left|\dot{\tilde{\sigma}}_{i}\right|=\left|\dot{\sigma}_{i}\right| \leq b_{\dot{\sigma}_{i}}$ in $t \in\left(t_{j}, t_{j+1}\right]$, where $b_{\dot{\sigma}_{i}}$ is a unknown constant. Thus, the following inequality holds.

$$
\begin{aligned}
& \tilde{\sigma}_{i}^{2}\left(t_{j+1}\right)-\tilde{\sigma}_{i}^{+}\left(t_{j}\right)^{2} \leq 2 b_{\dot{\sigma}_{i}} T_{\max }\left|\tilde{\sigma}_{i}^{+}\left(t_{j}\right)\right|+b_{\dot{\sigma}_{i}}^{2} T_{\max }^{2} \\
& \leq 2 b_{\dot{\sigma}} T_{\max } \sqrt{V_{i}+\Delta V_{i}}+b_{\dot{\sigma}_{i}}^{2} T_{\max }^{2}
\end{aligned}
$$

Thus, a new interconnected system is obtained as

$$
\left\{\begin{array}{l}
\Delta V_{i} \leq-a_{i} V_{i}+b_{i} \\
\Theta V_{i} \leq-a_{i} V_{i}+2 b_{\dot{\sigma}_{i}} T_{\max } \sqrt{V_{i}+\Delta V_{i}}+b_{\dot{\sigma}_{i}}^{2} T_{\max }^{2}+b_{i}
\end{array}\right.
$$

If $V_{i}\left(t_{j}\right)>b_{i} / a_{i}$, it has $\Delta V_{i}<0$ for the first inequality in (A-5). In this case, the second inequality in (A-5) is transformed to $\Theta V_{i} \leq-a_{i} V_{i}+2 b_{\dot{\sigma}_{i}} T_{\max } \sqrt{V_{i}}+b_{\dot{\sigma}_{i}}^{2} T_{\max }^{2}+$ $b_{i}$. By solving this inequality, it has $\Theta V_{i}<0$ for $V_{i}\left(t_{j}\right)>$ $\left(b_{\dot{\sigma}_{i}} T_{\max }+\sqrt{b_{\dot{\sigma}_{i}}^{2} T_{\max }^{2}\left(1+a_{i}\right)+a_{i} b_{i}}\right)^{2} / a_{i}^{2}$. Thus, it is concluded that $\Theta V_{i}<0$ for $V_{i}\left(t_{j}\right)>\max \left\{b_{i} / a_{i},\left(b_{\dot{\sigma}_{i}} T_{\max }+\right.\right.$ $\left.\left.\sqrt{b_{\dot{\sigma}_{i}}^{2} T_{\max }^{2}\left(1+a_{i}\right)+a_{i} b_{i}}\right)^{2} / a_{i}^{2}\right\}$. Because $V_{i}^{+}\left(t_{j}\right)<V_{i}(t)<$ $V_{i}\left(t_{j+1}\right)$ in $t \in\left(t_{j}, t_{j+1}\right]$, it is inferred that $V_{i}(t)$ is bounded all along and is ultimately bounded by $\max \left\{b_{i} / a_{i},\left(b_{\dot{\sigma}_{i}} T_{\text {max }}+\right.\right.$ $\left.\left.\sqrt{b_{\dot{\sigma}_{i}}^{2} T_{\max }^{2}\left(1+a_{i}\right)+a_{i} b_{i}}\right)^{2} / a_{i}^{2}\right\}$. The proof is completed.

It is obvious that the larger $T_{\max }$ (namely by increasing $\mu$ ) will lead to the smaller $a_{i}$ and the larger ultimate bound of $V_{i}$, which suggests the slower learning rate and the worse identification accuracy.

\section{B Proof of Lemma 2}

For convenience, set $\zeta=1$ in (17). By subtracting $\left[\alpha_{i}, 0\right]^{\mathrm{T}}$ from (17), it renders

$\left[\begin{array}{c}\dot{q}_{i} \\ \dot{\eta}_{i}\end{array}\right]=\left[\begin{array}{cc}0 & 1 \\ -\omega^{2} & -2 \omega\end{array}\right]\left[\begin{array}{c}q_{i} \\ \eta_{i}\end{array}\right]-\left[\begin{array}{c}\dot{\alpha}_{i} \\ 0\end{array}\right]$

Define $A=\left[0,1 ;-\omega^{2},-2 \omega\right]$. It is calculated that $A$ has two repeated eigenvalues of $\lambda(A)=-\omega$. Through the HamiltonCayley theorem, it can be derived that

$\left\{\begin{array}{l}a_{1}(t) \lambda(A)+a_{0}(t)=e^{\lambda(A) t} \\ a_{1}(t)=t e^{\lambda(A) t}\end{array}\right.$

Thus, $a_{0}(t)=(1+\omega t) e^{-\omega t}$ and $a_{1}(t)=t e^{-\omega t}$ are obtained. Ulteriorly, the state transformation matrix of (B-1) in $t \in$ $\left(t_{j}, t_{j+1}\right]$ is obtained as $\Phi(t)=a_{1}\left(t-t_{j}\right) A+a_{0}\left(t-t_{j}\right)$. Then, the analytical solution of (B-1) is derived as

$\left[\begin{array}{c}q_{i}(t) \\ \eta_{i}(t)\end{array}\right]=\Phi(t)\left[\begin{array}{c}q_{i}^{+}\left(t_{j}\right) \\ \eta_{i}\left(t_{j}\right)\end{array}\right]+\int_{t_{j}}^{t} \Phi(\tau)\left[\begin{array}{c}\dot{\alpha}_{i}\left(t-t_{j}-\tau\right) \\ 0\end{array}\right] \mathrm{d} \tau$

Because all the states are defined in a compact set, it is tenable to assume $\left|\dot{\alpha}_{i}(t)\right| \leq b_{\dot{\alpha}_{i}}$, where $b_{\dot{\alpha}_{i}}$ is an unknown con- stant. Then, the following inequality can be derived from (B$3)$.

$$
\left\{\begin{aligned}
\left|q_{i}(t)\right| \leq & \left|q_{i}^{+}\left(t_{j}\right)\right|\left(1+\omega\left(t-t_{j}\right)\right) e^{-\omega\left(t-t_{j}\right)} \\
& +\left(\frac{2}{\omega}-\frac{2}{\omega} e^{-\omega\left(t-t_{j}\right)}-\left(t-t_{j}\right) e^{-\omega\left(t-t_{j}\right)}\right) b_{\dot{\alpha}_{i}} \\
& +\left|\eta_{i}\left(t_{j}\right)\right|\left(t-t_{j}\right) e^{-\omega\left(t-t_{j}\right)} \\
\left|\eta_{i}(t)\right| \leq & \left|q_{i}^{+}\left(t_{j}\right)\right| \omega^{2}\left(t-t_{j}\right) e^{-\omega\left(t-t_{j}\right)} \\
& +\left|\left(1-\omega\left(t-t_{j}\right)\right) e^{-\omega\left(t-t_{j}\right)}\right| \times\left|\eta_{i}\left(t_{j}\right)\right| \\
& +\left(1-e^{-\omega\left(t-t_{j}\right)}-w\left(t-t_{j}\right) e^{-\omega\left(t-t_{j}\right)}\right) b_{\dot{\alpha}_{i}}
\end{aligned}\right.
$$

For (B-4), an assumption is made at first that $\left|q_{i}^{+}\left(t_{j}\right)\right| \leq$ $\mu_{q_{i}}+b_{q_{i}}$ and $\left|\eta_{i}\left(t_{j}\right)\right| \leq \mu_{\eta_{i}}+b_{\dot{\alpha}_{i}}$, where $\mu_{q_{i}}$ and $\mu_{\eta_{i}}$ can two arbitrarily small positive constants. For the first inequality in (B-4), it is inferred that $\left|q_{i}(t)\right| \rightarrow 0$ with $\omega \rightarrow+\infty$. Thus, there must exist $\omega=\omega_{q_{i}}$ such that $\left|q_{i}\left(t_{j}+\delta_{t}\right)\right| \leq \mu_{q_{i}}$ holds, where $\delta_{t}$ is the minimum inter-event time proved in Section IV. Invoking $\left|\Delta q_{i}\right| \leq b_{q_{i}},\left|q_{i}^{+}\left(t_{j}\right)\right| \leq \mu_{q_{i}}+b_{q_{i}}$ holds. For the second inequality in (B-4), it is inferred that $\left|\eta_{i}(t)\right| \leq b_{\dot{\alpha}_{i}}$ with $\omega \rightarrow+\infty$. Thus, there must exist $\omega=\omega_{\eta_{i}}$ such that $\mid \eta_{i}\left(t_{j}+\right.$ $\left.\delta_{t}\right) \mid \leq \mu_{\eta_{i}}+b_{\dot{\alpha}_{i}}$ holds. By selecting $\omega=\max \left\{\omega_{q_{i}}, \omega_{\eta_{i}}\right\}$, this assumption holds.

From the first inequality in (B-4), it can be found that $\left|\left(1+\omega\left(t-t_{j}\right)\right) e^{-\omega\left(t-t_{j}\right)}\right|<1, \mid 2 / \omega-2 / \omega e^{-\omega\left(t-t_{j}\right)}-(t-$ $\left.t_{j}\right) e^{-\omega\left(t-t_{j}\right)} \mid<2 / \omega$ and $\left|\left(t-t_{j}\right) e^{-\omega\left(t-t_{j}\right)}\right|<1 /(\omega e)$. By invoking the above assumption, the first inequality in (B-4) can be transformed to

$\left|q_{i}(t)\right| \leq \mu_{q_{i}}+b_{q_{i}}+\frac{\mu_{\eta_{i}}+b_{\dot{\alpha}_{i}}}{\omega e}+\frac{2 b_{\dot{\alpha}_{i}}}{\omega}$

Let $\bar{b}_{\underline{q}_{i}}=\mu_{q_{i}}+b_{q_{i}}+\left(\mu_{\eta_{i}}+b_{\dot{\alpha}_{i}}\right) /(\omega e)+2 b_{\dot{\alpha}_{i}} / \omega$. It is obvious that $\bar{b}_{q_{i}} \rightarrow b_{q_{i}}$ with $\omega \rightarrow+\infty$. The proof is completed.

Acknowledgements This work is supported by the Foundation for Innovative Research Groups of the Natural Science Foundation of Hebei Province (No.E2020203174) and Hebei Province Natural Science Fund (No.E2019203431) titled with "Research on Multi-Field Coupling Dynamics Modeling for Rescue Helicopter Simulator". We'd like to thank Professor Chen Bing in Institute of Complexity Science, Qingdao University for his suggestions on the nonstrict-nonlinear system.

\section{Compliance with ethical standards}

Conflict of interest There is no potential conflict of interest in this paper.

\section{References}

1. Tong, S., Li, Y., Sui, S.: Adaptive fuzzy output feedback control for switched nonstrict-feedback nonlinear systems with input nonlinearities. IEEE Transactions on Fuzzy Systems 24(6), 1426-1440 (2016)

2. Tong, S., Li, Y., Sui, S.: Adaptive fuzzy tracking control design for siso uncertain nonstrict feedback nonlinear systems. IEEE Transactions on Fuzzy Systems 24(6), 1441-1454 (2016) 
3. Chen, B., Liu, X.P., Ge, S.S., Lin, C.: Adaptive fuzzy control of a class of nonlinear systems by fuzzy approximation approach. IEEE Transactions on Fuzzy Systems 20(6), 1012-1021 (2012)

4. Chen, B., Liu, K., Liu, X., Shi, P., Lin, C., Zhang, H.: Approximation-based adaptive neural control design for a class of nonlinear systems. IEEE Transactions on $\mathrm{Cy}-$ bernetics 44(5), 610-619 (2014)

5. Chen, B., Zhang, H., Lin, C.: Observer-based adaptive neural network control for nonlinear systems in nonstrictfeedback form. IEEE Transactions on Neural Networks and Learning Systems 27(1), 89-98 (2016)

6. Zhou, Q., Li, H., Wu, C., Wang, L., Ahn, C.K.: Adaptive fuzzy control of nonlinear systems with unmodeled dynamics and input saturation using small-gain approach. IEEE Transactions on Systems, Man, and Cybernetics: Systems 47(8), 1979-1989 (2017)

7. Wang, H., Liu, X., Liu, K., Karimi, H.R.: Approximationbased adaptive fuzzy tracking control for a class of nonstrict-feedback stochastic nonlinear time-delay systems. IEEE Transactions on Fuzzy Systems 23(5), 1746$1760(2015)$

8. Wang, H., Liu, K., Liu, X., Chen, B., Lin, C.: Neuralbased adaptive output-feedback control for a class of nonstrict-feedback stochastic nonlinear systems. IEEE Transactions on Cybernetics 45(9), 1977-1987 (2015)

9. Zhou, Q., Li, H., Wang, L., Lu, R.: Prescribed performance observer-based adaptive fuzzy control for nonstrict-feedback stochastic nonlinear systems. IEEE Transactions on Systems, Man, and Cybernetics: Systems 48(10), 1747-1758 (2018)

10. Li, Y., Tong, S.: Command-filtered-based fuzzy adaptive control design for mimo-swithced nonstrict-feedback nonlinear systems. IEEE Transactions on Fuzzy Systems 25(3), 668-681 (2017)

11. Li, Y., Li, K., Tong, S.: Finite-time adaptive fuzzy output feedback dynamic surface control for mimo nonstrict feedback systems. IEEE Transactions on Fuzzy Systems 27(1), 96-110 (2019)

12. Liu, Y., Li, S., Tong, S., Chen, C.L.P.: Neural approximation-based adaptive control for a class of nonlinear nonstrict feedback discrete-time systems. IEEE Transactions on Neural Networks and Learning Systems 28(7), 1531-1541 (2017)

13. Ge, S.S., Li, G.Y., Lee, T.H.: Adaptive nn control for a class of strict-feedback discrete-time nonlinear systems. Automatica 39(5), 807-819 (2003)

14. Bai, W., Li, T., Tong, S.: Nn reinforcement learning adaptive control for a class of nonstrict-feedback discretetime systems. IEEE Transactions on Cybernetics 50(11), 4573-4584 (2020)

15. Ioannou, P., Sun, J.: Robust adaptive control. Prentice Hall, Upper Saddle River (1996)

16. Pan, Y., Zhou, Y., Sun, T., Er, M.J.: Composite adaptive fuzzy $h_{\infty}$ tracking control of uncertain nonlinear systems. Neurocomputing 99, 15-24 (2013)

17. Xu, B., Shi, Z., Yang, C., Sun, F.: Composite neural dynamic surface control of a class of uncertain nonlinear systems in strict-feedback form. IEEE Transactions on Cybernetics 44(12), 2626-2634 (2014)

18. Li, Y., Tong, S.: Composite adaptive fuzzy output feedback control design for uncertain nonlinear strictfeedback systems with input saturation. IEEE Transactions on Cybernetics 45, 2299-2308 (2015)

19. Li, Y., Tong, S., Li, T.: Hybrid fuzzy adaptive output feedback control design for uncertain mimo nonlinear systems with time-varying delays and input saturation. IEEE Transactions on Fuzzy Systems 24, 841-853 (2016)

20. Xu, B., Sun, F., Pan, Y., Chen, B.: Disturbance observer based composite learning fuzzy control of nonlinear systems with unknown dead zone. IEEE Transactions on Systems, Man, and Cybernetics: Systems 47(8), 1854$1862(2017)$

21. Xu, B.: Composite learning control of flexible-link manipulator using nn and dob. IEEE Transactions on Systems, Man, and Cybernetics: Systems 48(11), 1979-1985 (2018)

22. Wang, L., Basin, M.V., Li, H., Lu, R.: Observer-based composite adaptive fuzzy control for nonstrict-feedback systems with actuator failures. IEEE Transactions on Fuzzy Systems 26(4), 2336-2347 (2018)

23. Deng, Y., Zhang, X., Zhang, Q., Hu, Y.: Event-triggered composite adaptive fuzzy control of sailboaat with heeling constraint. Ocean Engineering 211, 107627 (2020)

24. Peng, Z., Wang, D., Wang, J.: Predictor-based neural dynamic surface control for uncertain nonlinear systems in strict-feedback form. IEEE Transactions on Neural Network and Learning Systems 28(9), 2156-2167 (2017)

25. Liu, L., Wang, D., Peng, Z.: Path following of marine surface vehicles with dynamical uncertainty and timevarying ocean disturbances. Neurocomputing 173, 799$808(2016)$

26. Pan, Y., Sun, T., Yu, H.: Composite adaptive dynamic surface control using online recorded data. International Journal of Robust and Nonlinear Control 26(18), 39213936 (2016)

27. Pan, Y., Yu, H.: Composite learning robot control with guaranteed parameter convergence. Automatica 89, 398$406(2018)$

28. Guo, K., Pan, Y., Zheng, D., Yu, H.: Composite learning control of robotic systems: a least squares modulated approach. Automatica, doi: 10.1016/j.automatica.2019.108612 (2020)

29. Xu, B., Yang, D., Shi, Z., Pan, Y., Chen, B., Sun, F.: Online recorded data-based composite neural control of strict-feedback systems with application to hypersonic flight dynamics. IEEE Transactions on Neural Networks and Learning Systems 29(8), 3839-3849 (2018)

30. Xu, B., Shou, Y., Luo, J., Pu, H., Shi, Z.: Neural learning control of strict-feedback systems using disturbance observer. IEEE Transactions on Neural Networks and Learning Systems 30(5), 1296-1307 (2019)

31. Xing, L., Wen, C., Liu, Z., Su, H., Cai, J.: Event-triggered adaptive control for a class of uncertain nonlinear systems. IEEE Transactions on Automatic Control 62(4), 2071-2076 (2017)

32. Cao, L., Li, H., Zhou, Q.: Adaptive intelligent control for nonlinear strict-feedback systems with virtual control coefficients and uncertain disturbances based on eventtriggered mechanism. IEEE Transactions on Cybernetics 48(12), 3390-3402 (2018)

33. Zhu, Z., Pan, Y., Zhou, Q., Lu, C.: Event-triggered adaptive fuzzy control for stochastic nonlinear systems with unmeasured states and unknown backlashlike hysteresis. IEEE Transactions on Fuzzy Systems, doi:10.1109/tfuzz.2020.2973950 (2020)

34. Sun, K., Qiu, J., Karimi, H.R., Fu, Y.: Eventriggered robust fuzzy adaptive finite-time control of nonlinear systems with prescribed performance. IEEE Transactions on Fuzzy Systems, doi: 10.1109/tfuzz.2020.2979129 (2020)

35. Wang, M., Wang, Z., Chen, Y., Sheng, W.: Eventbased adaptive neural tracking control for discrete-time 
stochastic nonlinear systems: a triggering threshold compensation strategy. IEEE Transactions on Neural Networks and Learning Systems 31(6), 1968-1981 (2020)

36. Wang, M., Wang, Z., Chen, Y., Sheng, W.: Adaptive neural event-triggered control for discrete-time strictfeedback nonlinear systems. IEEE Transactions on Cybernetics 50(7), 2946-2958 (2020)

37. Szanto, N., Narayanan, V., Jagannathan, S.: Eventsampled direct adaptive nn output-and state-feedback control of uncertain strict-feedback system. IEEE Transactions on Neural Networks and Learning Systems 29(5), 1850-1863 (2018)

38. Li, Y., Yang, G.: Model-based adaptive event-triggered control of strict-feedback nonlinear systems. IEEE Transactions on Neural Networks and Learning Systems 29(4), 1033-1045 (2018)

39. Li, Y., Yang, G.: Observer-based fuzzy adaptive eventtriggered control co-design for a class of uncertain nonlinear systems. IEEE Transactions on Fuzzy Systems 26(3), 1589-1599 (2018)

40. Cao, L., Li, H., Wang, N., Zhou, Q.: Observer-based event-triggered adaptive decentralized fuzzy control for nonlinear large-scale systems. IEEE Transactions on Fuzzy Systems 27(6), 1201-1214 (2019)

41. Ma, J., Xu, S., Zhang, Z.: Event-triggered adaptive neural network control for nonstrict-feedback nonlinear timedelay systems with unknown control directions. IEEE Transactions on Neural Networks and Learning Systems, doi:10.1109/tnnls.2019.2952709 31(10), 4196-4205 (2020)

42. Xu, Y., Zhou, Q., Li, T., Liang, H.: Event-triggered neural control for non-strict-feedback systems with actuator failures. IET Control Theory \& Applications 13(2), 171$182(2019)$

43. Wang, A., Liu, L., Qiu, J., Feng, G.: Finite-time adaptive fuzzy control for nonstrict-feedback nonlinear systems via an event-triggered strategy. IEEE Transactions on Fuzzy Systems 28(9), 2164-2174 (2020)

44. Wang, A., Liu, L., Qiu, J.: Event-triggered adaptive fuzzy output-feedback control for nonstrict-feedback nonlinear systems with asymmetric output constraint. IEEE Transactions on Cybernetics, doi:10.1109/tcyb.2020.2974775 (2020)

45. Wang, W., Li, Y., Tong, S.: Neural-networkbased adaptive event-triggered consensus control of nonstrict-feedback nonlinear systems. IEEE Transactions on Neural Networks and Learning Systems, doi:10.1109/tnnls.2020.2991015 (2020)

46. Wang, A., Liu, L., Qiu, J., Feng, G.: Event-triggered robust adaptive fuzzy control for a class of nonlinear systems. IEEE Transactions on Fuzzy Systems 27(8), 1648$1658(2019)$

47. Sahoo, A., Xu, H., Jagannathan, S.: Neural networkbased event-triggered state feedback control of nonlinear continuous-time systems. IEEE Transactions on Neural Networks and Learning Systems 27(3), 497-509 (2016)

48. Narayanan, V., Jagannathan, S., Ramkumar, K.: Eventsampled output feedback control of robot manipulators using neural networks. IEEE Transactions on Neural Networks and Learning Systems 30(6), 1651-1658 (2019)

49. Deng, Y., Zhang, X., Im, N., Zhang, G., Zhang, Q.: Event-triggered robust fuzzy path following control for underactuated ships with input saturation. Ocean Engineering 186, 106122 (2019)

50. Deng, Y., Zhang, X., Zhang, G., Huang, C.: Parallel guidance and event-triggered robust fuzzy control for path following of autonomous wing-sailed catamaran. Ocean Engineering 190, 106442 (2019)

51. Jiao, J., Wang, G.: Event triggered trajectory tracking control approach for fully actuated surface vessel. Neurocomputing 182, 267-273 (2016)

52. Deng, Y., Zhang, X., Im, N., Zhang, G., Zhang, Q.: Model-based event-triggered tracking control of underactuated surface vessels with minimum learning parameters. IEEE Transactions on Neural Networks and Learning Systems 31(10), 4001-4014 (2020)

53. Deng, Y., Zhang, X.: Event-triggered composite adaptive fuzzy output feedback control for path following of autonomous surface vessels. IEEE Transactions on Fuzzy Systems, doi:10.1109/tfuzz.2020.3006562 (2020)

54. Farrell, J.A., Polycarpou, M., Sharma, M., Dong, W.: Command filtered backstepping. IEEE Transactions on Automatic Control 54(6), 1391-1395 (2009)

55. Chang, Y., Chan, W.: Adaptive dynamic surface control for uncertain nonlinear systems with interval type-2 fuzzy neural networks. IEEE Transactions on Cybernetics 44(2), 293-304 (2014) 
Figures

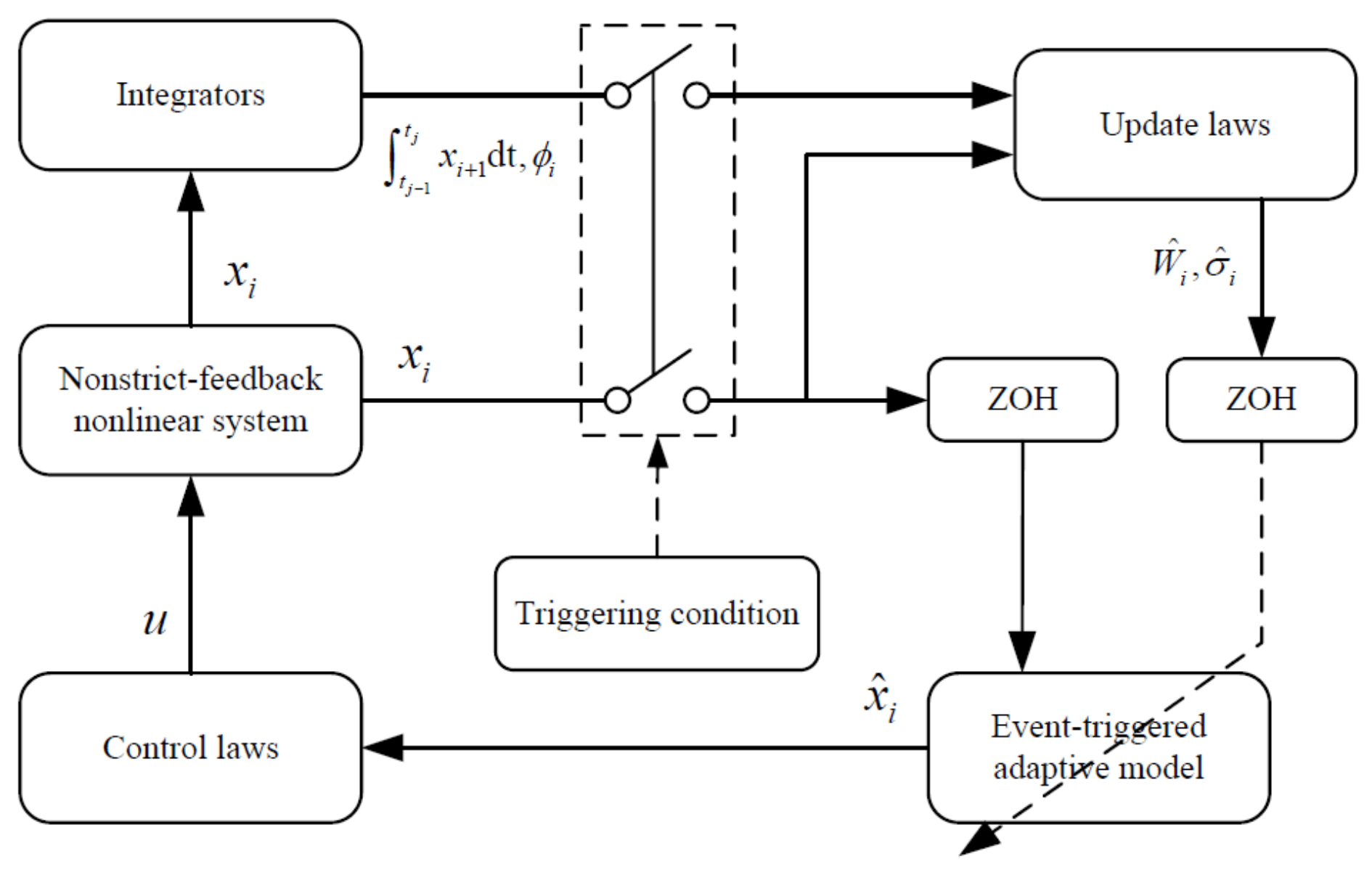

Figure 1

Framework of proposed ETC 


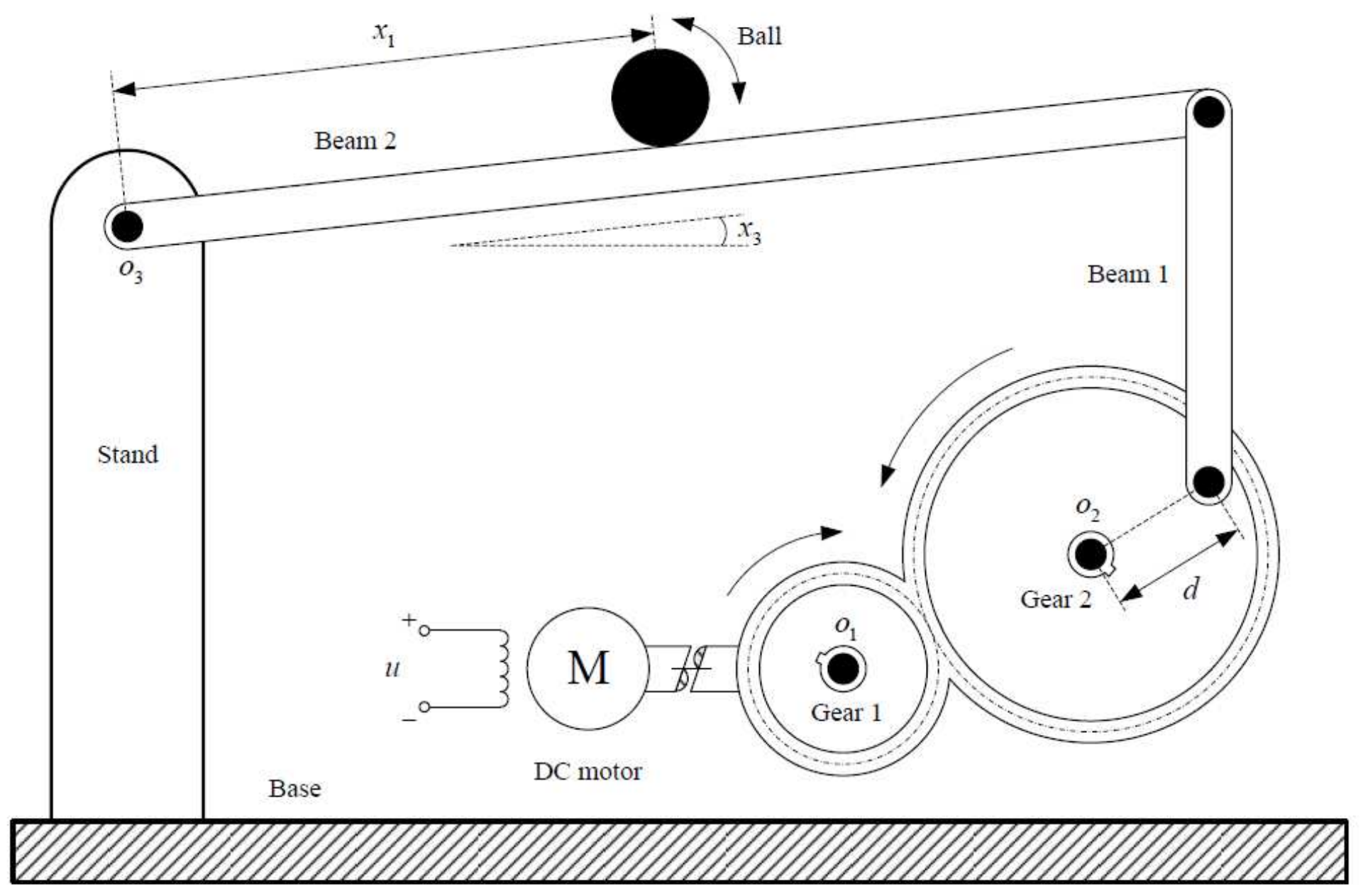

Figure 2

Diagram of ball and beam system 


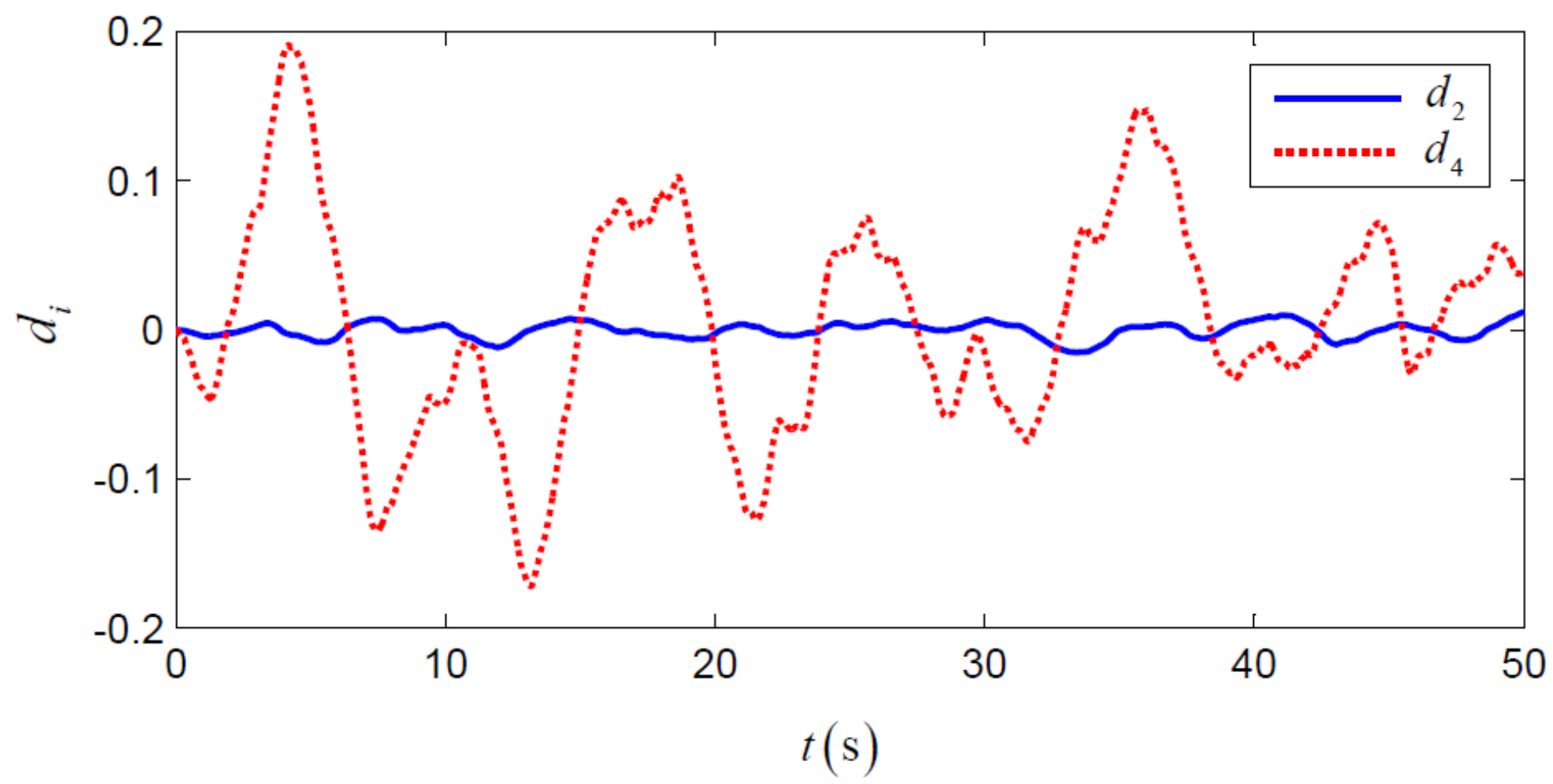

Figure 3

Disturbance di

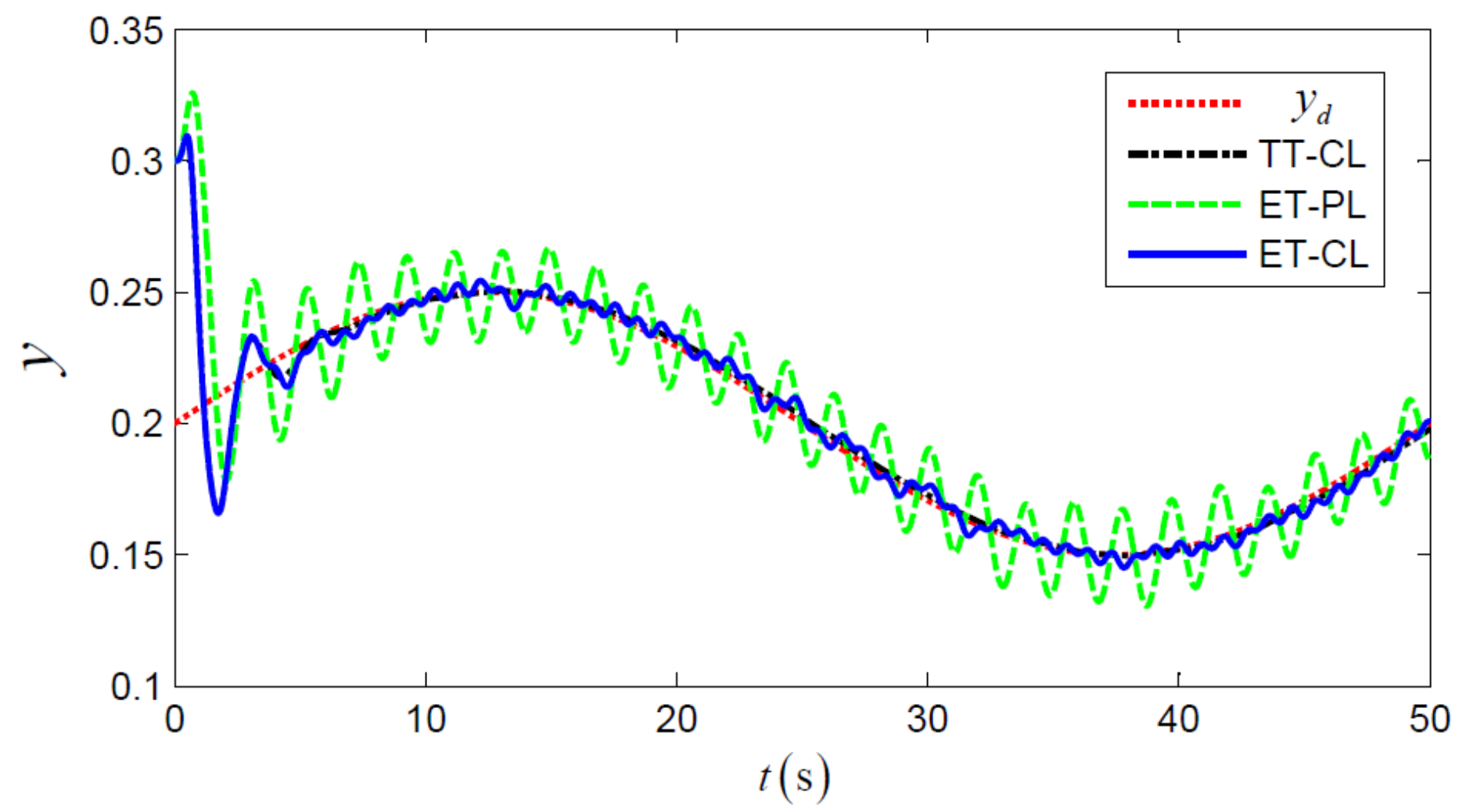

Figure 4 
Output y
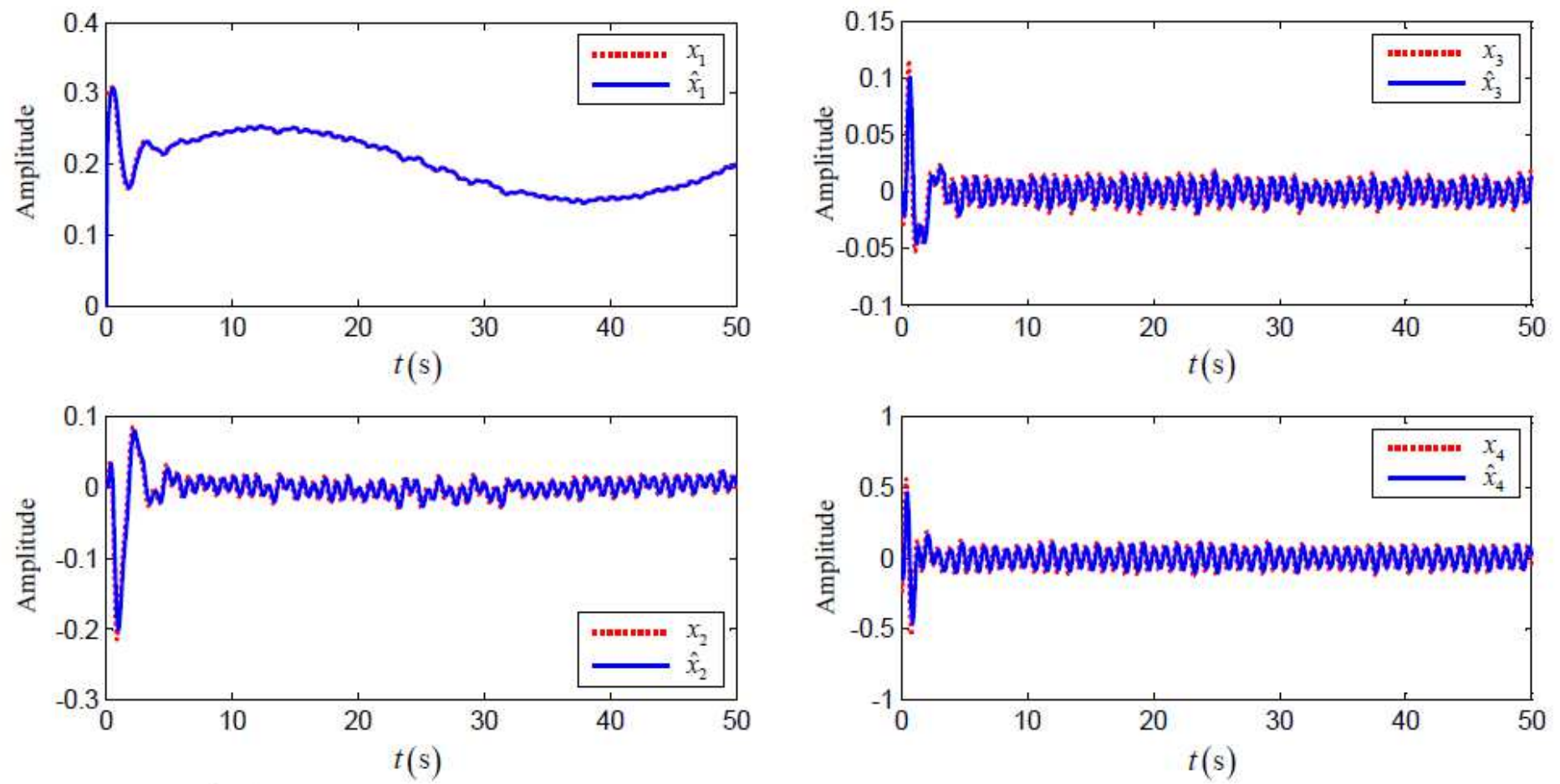

(a) $\hat{x}_{1}$ and $\hat{x}_{2}$

(b) $\hat{x}_{3}$ and $\hat{x}_{4}$

Figure 5

States of event-triggered adaptive model in ET-CL

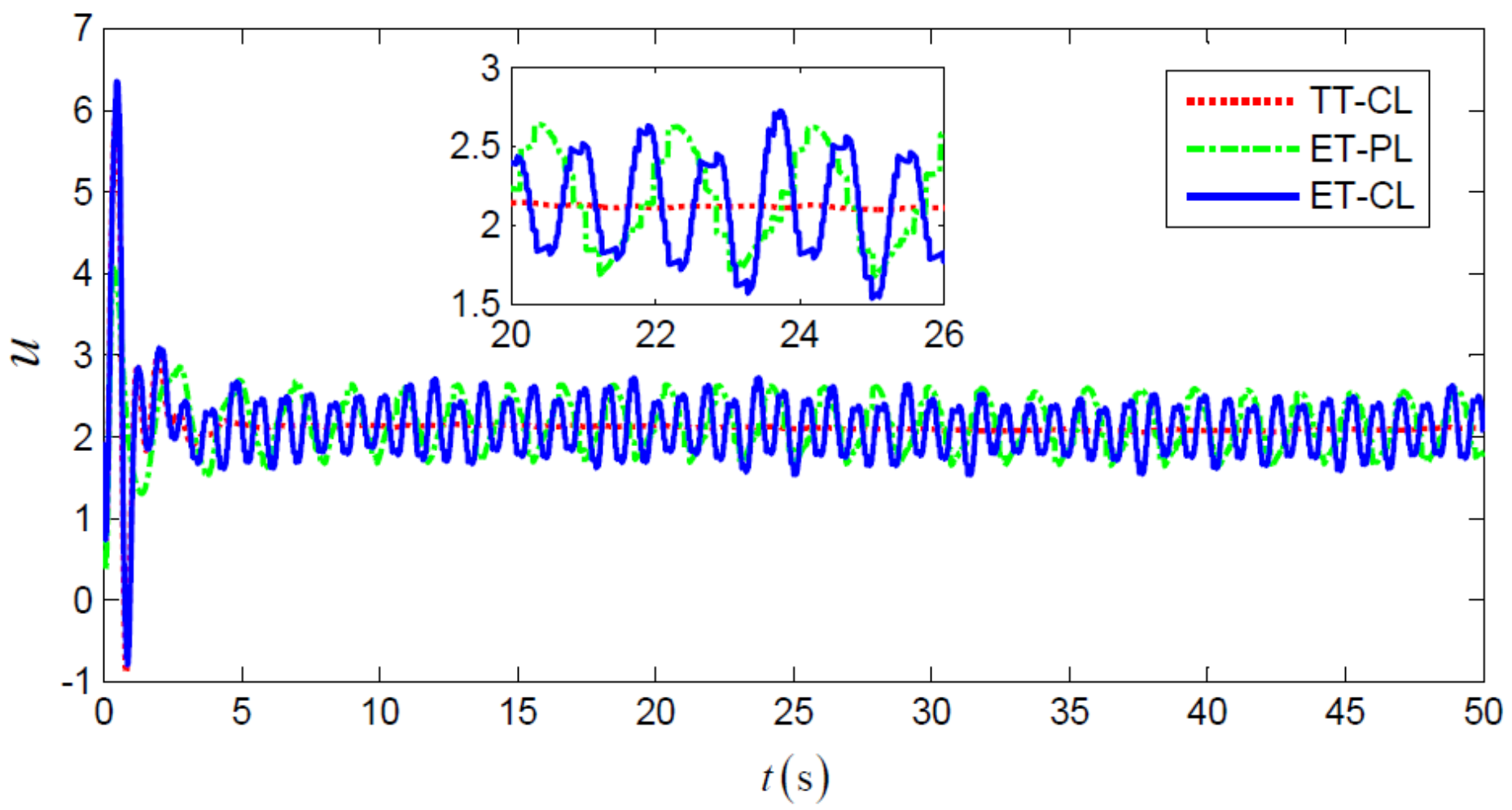


Figure 6

Control input u
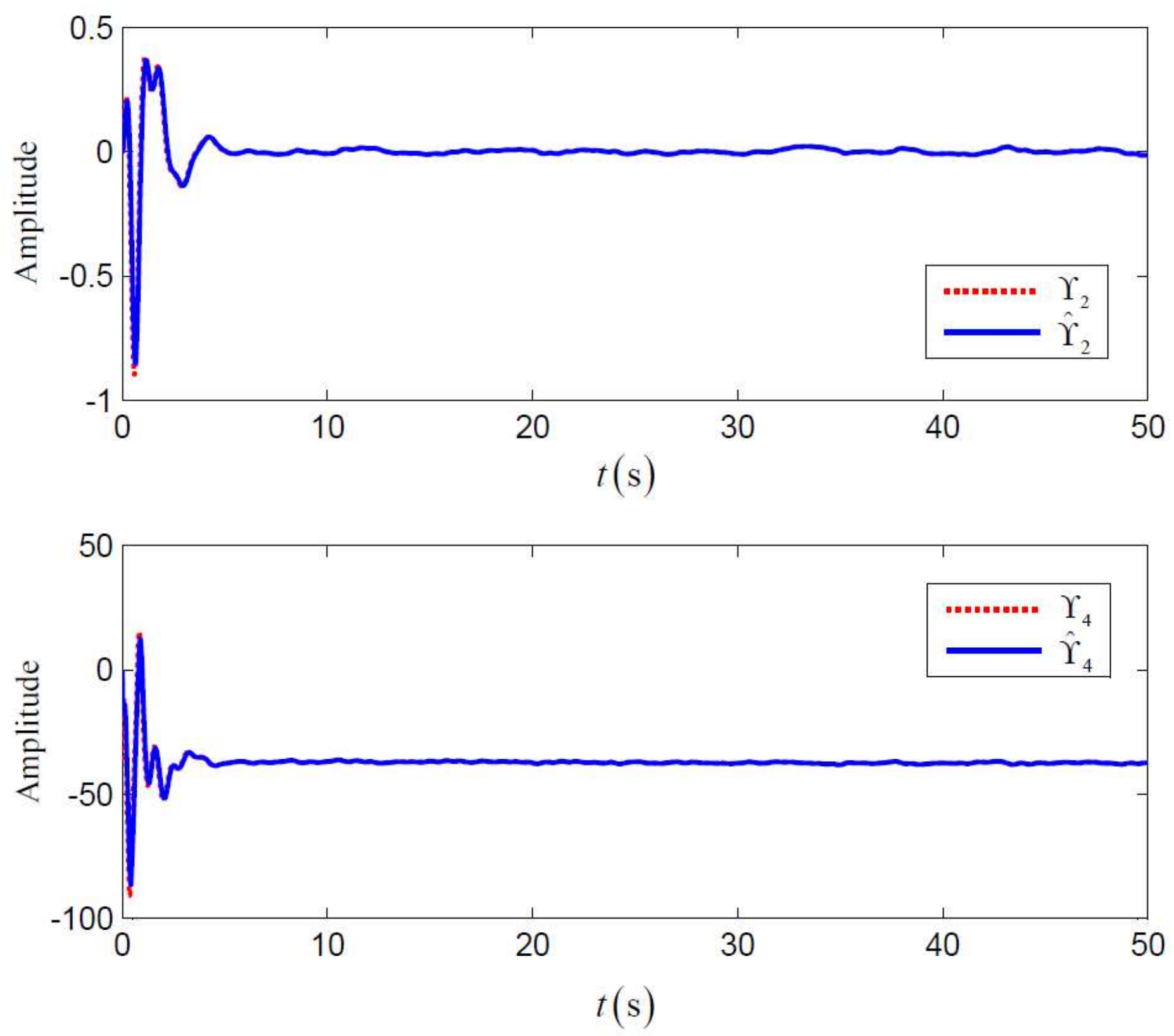

Figure 7

Learning performance of TT-CL 

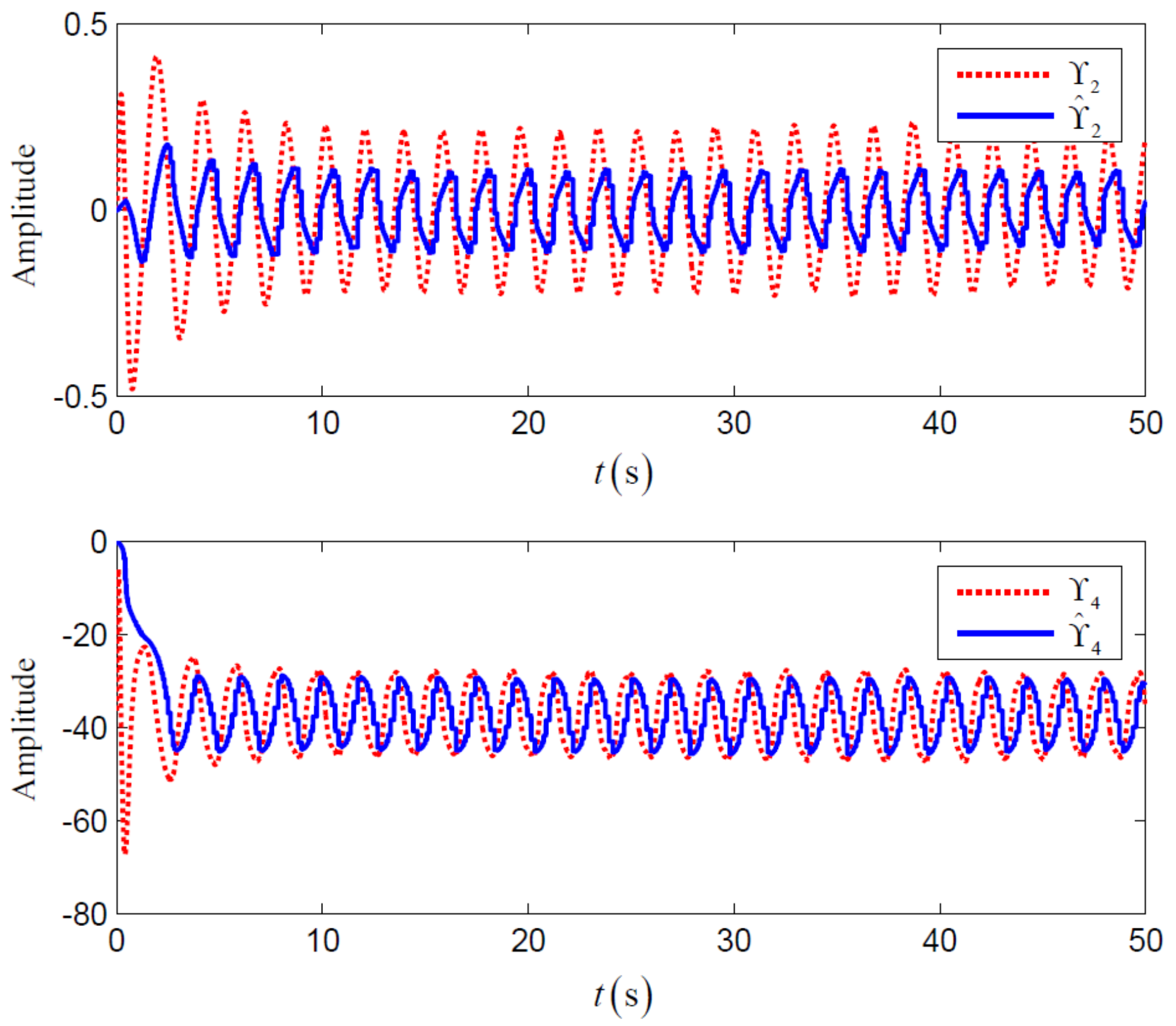

Figure 8

Learning performance of ET-PL 

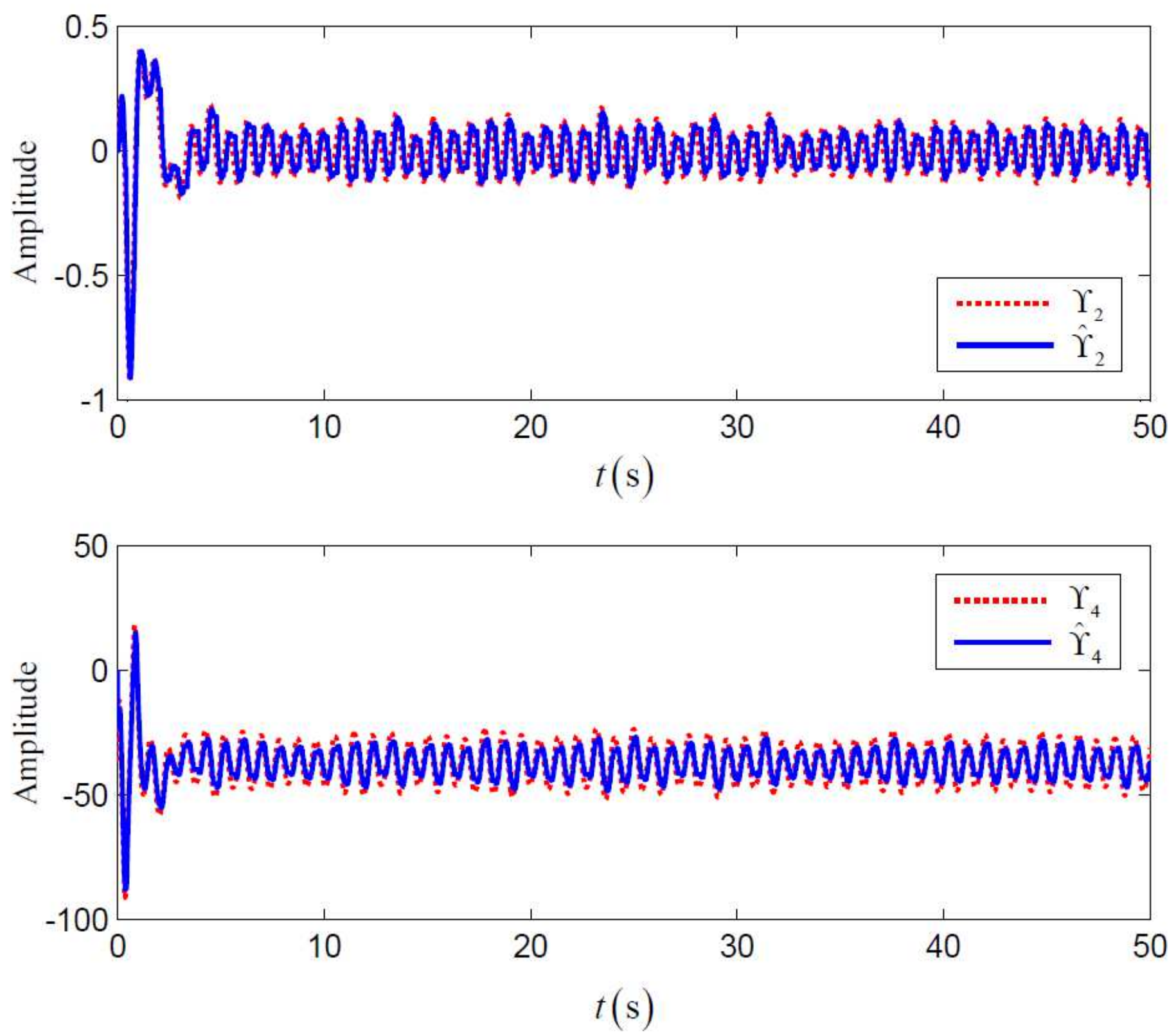

Figure 9

Learning performance of ET-CL 

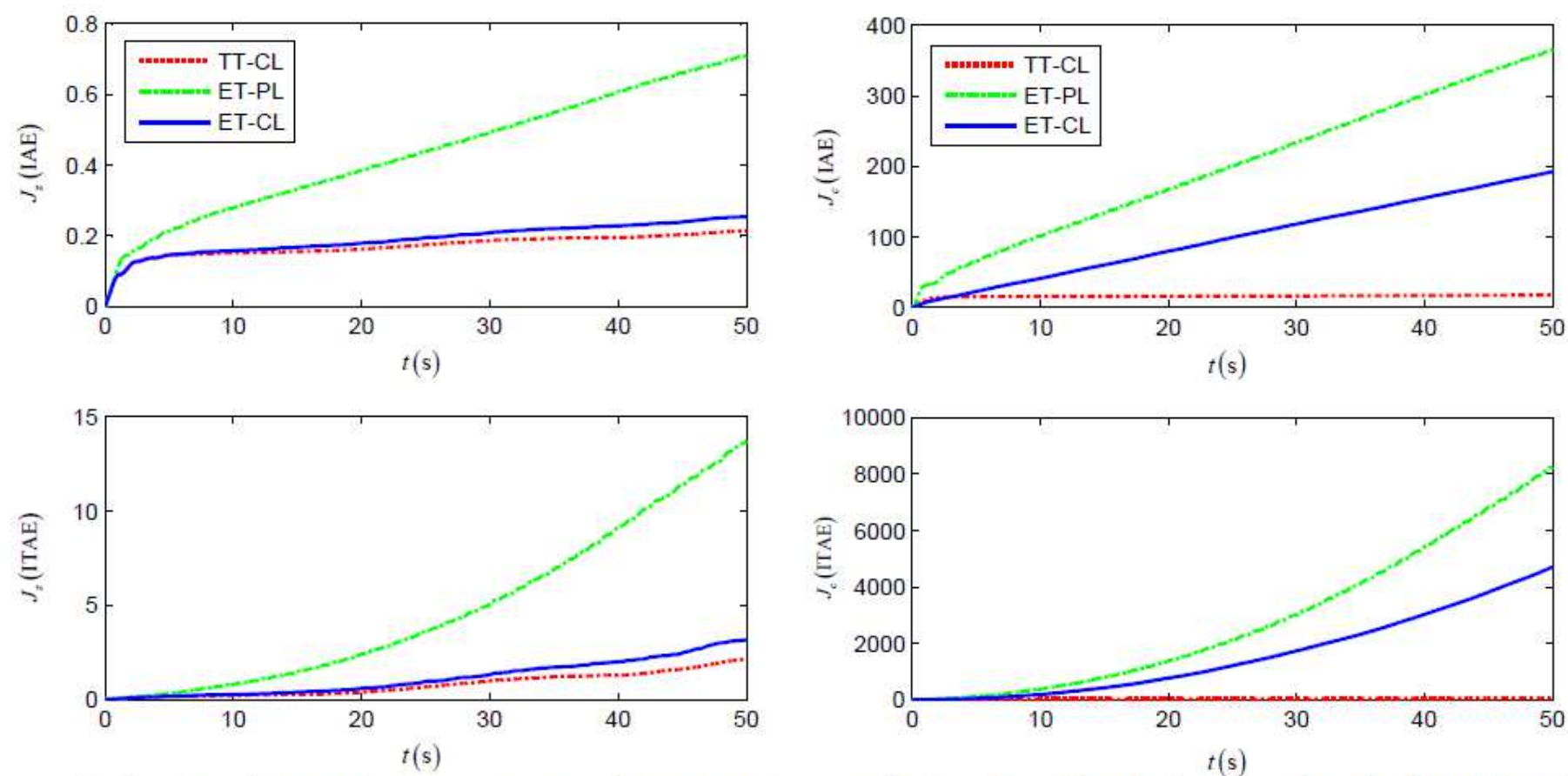

(a) $J_{z}(\mathrm{IAE})$ and $J_{z}$ (ITAE)

(b) $J_{c}$ (IAE) and $J_{c}$ (ITAE)

Figure 10

Evolution of performance indexes
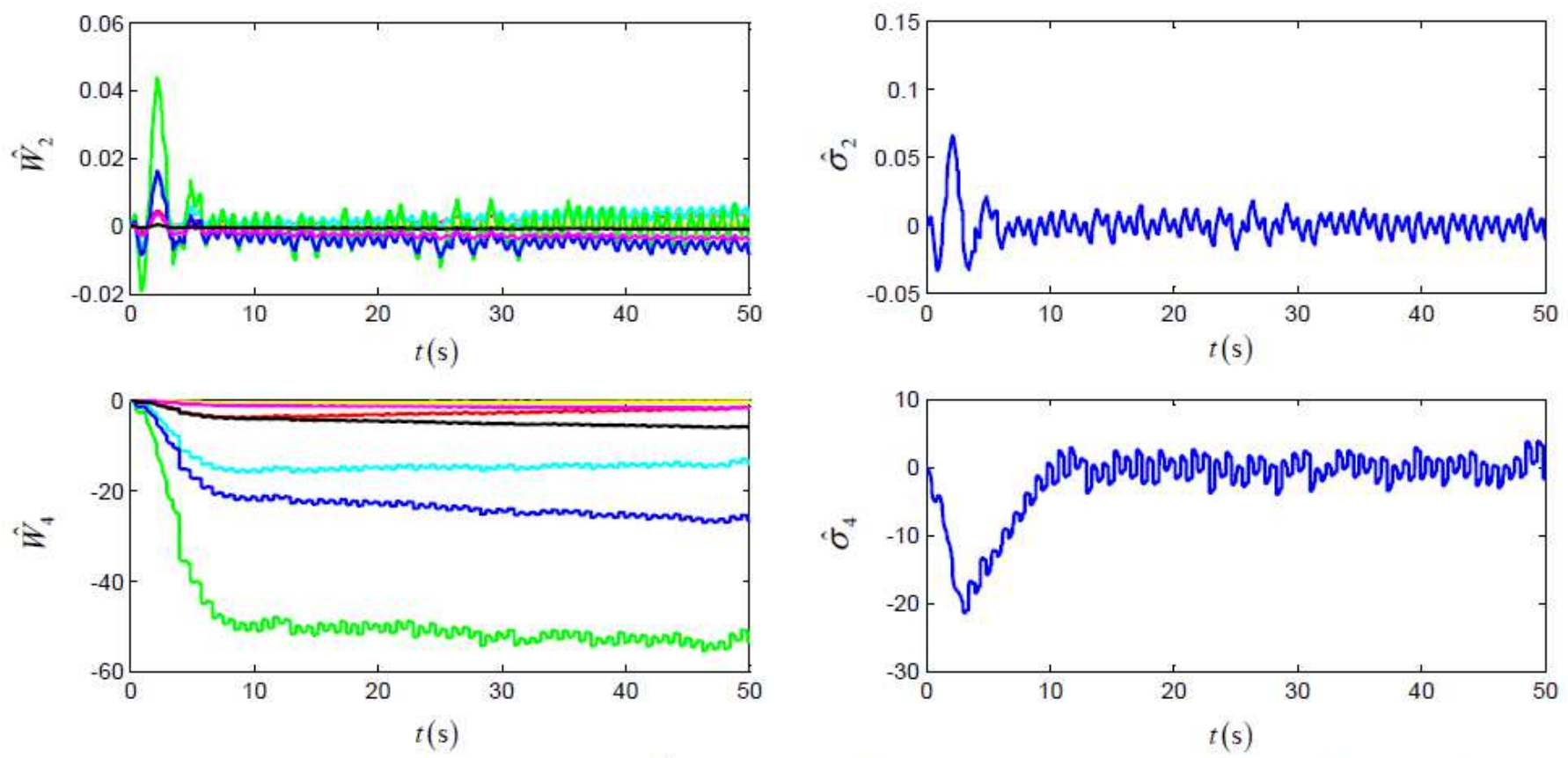

(a) Fuzzy weights $\hat{W}_{i}$

(b) Compound disturbance $\hat{\sigma}_{i}$ 
Figure 11

Update laws of ET-CL

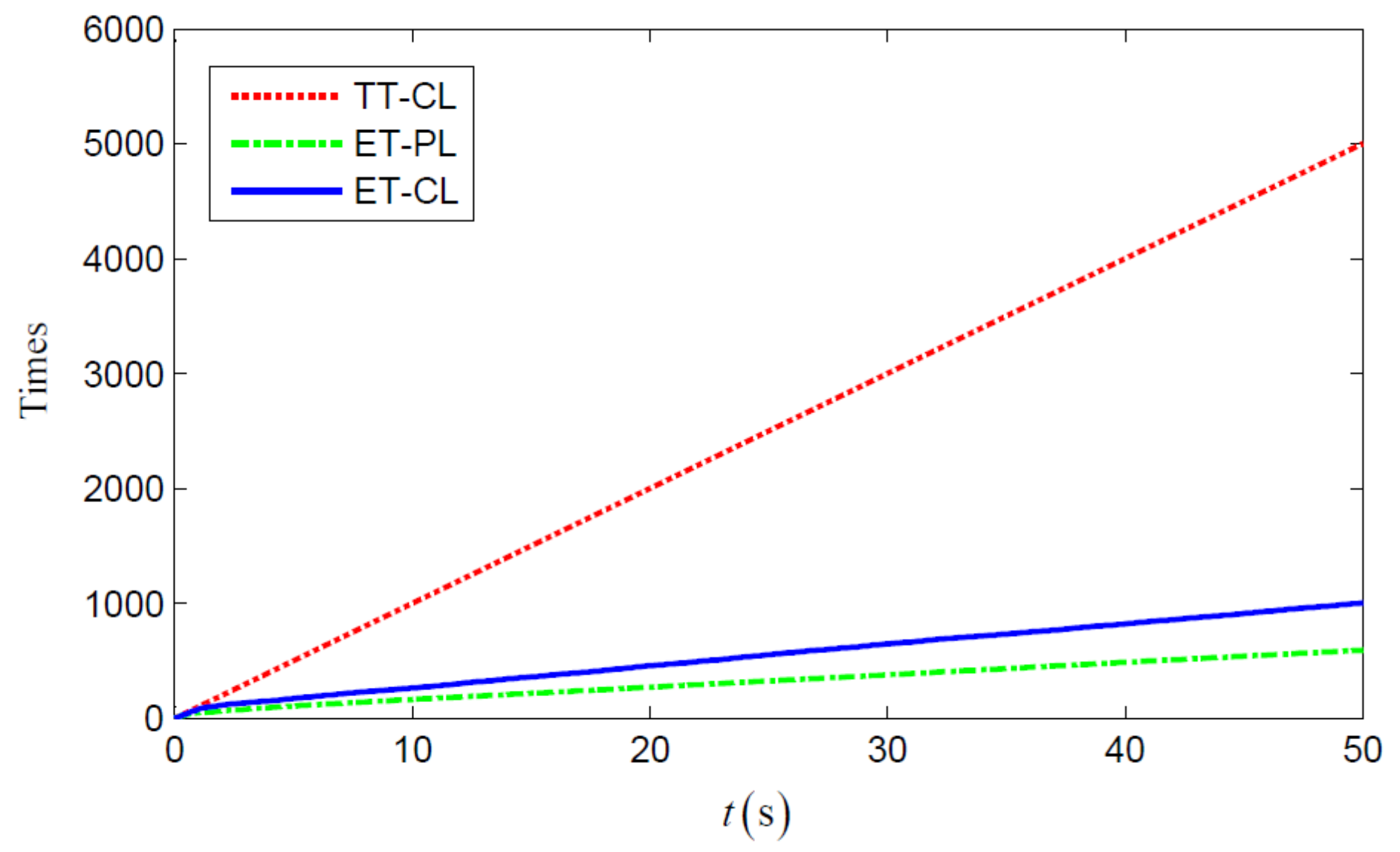

Figure 12

Accumulation of sampling times 


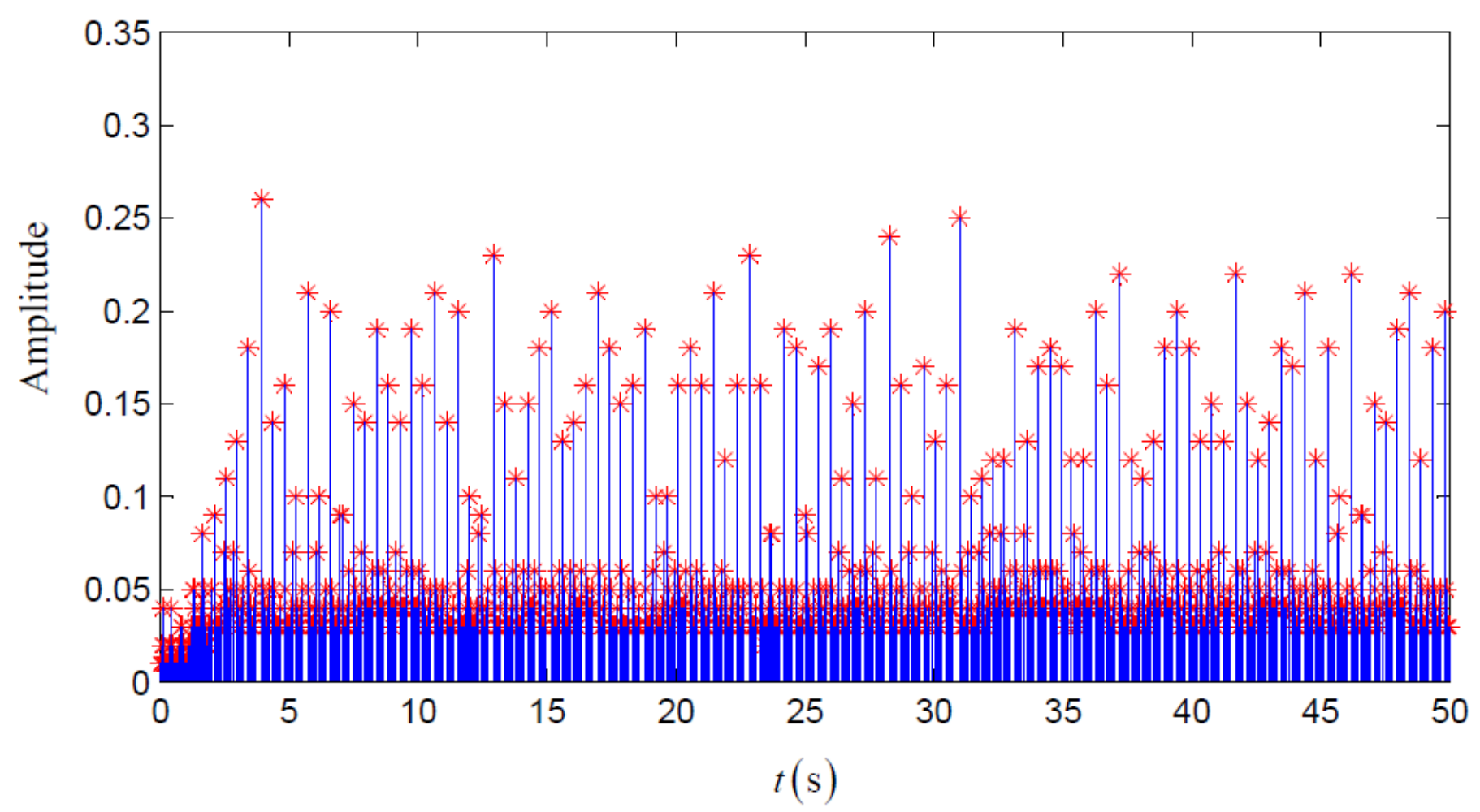

Figure 13

Inter-event time of ET-CL 